\title{
CONTRACTIVITY AND COMPLETE CONTRACTIVITY FOR FINITE DIMENSIONAL BANACH SPACES
}

\author{
GADADHAR MISRA, AVIJIT PAL AND CHERIAN VARUGHESE
}

\begin{abstract}
Choose an arbitrary but fixed set of $n \times n$ matrices $A_{1}, \ldots, A_{m}$ and let $\Omega_{\mathbf{A}} \subset \mathbb{C}^{m}$ be the unit ball with respect to the norm $\|\cdot\|_{\mathbf{A}}$, where $\left\|\left(z_{1}, \ldots, z_{m}\right)\right\|_{\mathbf{A}}=\left\|z_{1} A_{1}+\cdots+z_{m} A_{m}\right\|_{\text {op }}$. It is known that if $m \geq 3$ and $\mathbb{B}$ is any ball in $\mathbb{C}^{m}$ with respect to some norm, say $\|\cdot\|_{\mathbb{B}}$, then there exists a contractive linear map $L:\left(\mathbb{C}^{m},\|\cdot\|_{\mathbb{B}}^{*}\right) \rightarrow \mathcal{M}_{k}$ which is not completely contractive. The characterization of those balls in $\mathbb{C}^{2}$ for which contractive linear maps are always completely contractive thus remains open. We answer this question for balls of the form $\Omega_{\mathbf{A}}$ in $\mathbb{C}^{2}$.
\end{abstract}

\section{Introduction}

In 1936 von Neumann (see [17, Corollary 1.2]) proved that if $T$ is a bounded linear operator on a separable complex Hilbert space $\mathcal{H}$, then, for all complex polynomials $p$,

$$
\|p(T)\| \leq\|p\|_{\infty, \mathbb{D}}:=\sup \{|p(z)|:|z|<1\}
$$

if and only if $\|T\| \leq 1$. Or, equivalently, the homomorphism $\rho_{T}$ induced by $T$ on the polynomial ring $P[z]$ by the rule $\rho_{T}(p)=p(T)$ is contractive if and only if $T$ is contractive.

The original proof of this inequality is intricate. A couple of decades later, Sz.-Nazy (see [17, Theorem 4.3]) proved that a bounded linear operator $T$ admits a unitary (power) dilation if and only if there exists a unitary operator $U$ on a Hilbert space $\mathcal{K} \supseteq \mathcal{H}$ such that

$$
P_{\mathcal{H}} p(U)_{\mid \mathcal{H}}=p(T)
$$

for all polynomials $p$. The existence of such a dilation may be established by actually constructing a unitary operator $U$ dilating $T$. This construction is due to Schaffer (cf. [14]). Clearly, the von Neumann inequality follows from the existence of a power dilation via the spectral theorem for unitary operators.

Let $P=\left(\left(p_{i j}\right)\right)$ be a $k \times k$ matrix valued polynomial in $m$ variables. Let

$$
\|P\|_{\infty, \Omega}=\sup \left\{\left\|\left(\left(p_{i j}(z)\right)\right)\right\|_{\mathrm{op}}: z \in \Omega\right\},
$$

where $\Omega \subseteq \mathbb{C}^{m}$ is a bounded open and connected set. Define $P(T)$ to be the operator $\left(\left(p_{i j}(T)\right)\right)$, $1 \leq i, j \leq k$. The homomorphism $\rho_{T}$ is said to be completely contractive if

$$
\|P(T)\| \leq\|P\|_{\infty, \Omega}, k=1,2, \ldots
$$

A deep theorem due to Arveson (cf. [1]) says that $T$ has a normal boundary dilation if and only if $\rho_{T}$ is completely contractive. Clearly, if $\rho_{T}$ is completely contractive, then it is contractive. The dilation theorems due to Sz.-Nazy and Ando (cf. [17]) give the non-trivial converse in the case of the disc and the bi-disc algebras.

However, Parrott (cf. [15]) showed that there are three commuting contractions for which it is impossible to find commuting unitaries dilating them. In view of Arveson's theorem this naturally leads to the question of finding other algebras $\mathcal{O}(\Omega)$ for which all contractive homomorphisms are necessarily completely contractive. At the moment, this is known to be true of the disc, bi-disc

\footnotetext{
The work of G.Misra was supported, in part, through the J C Bose National Fellowship and UGC-CAS. The work of A. Pal was supported, in part, through the UGC-NET and the IFCAM Research Fellowship. The results of this paper are taken from his PhD thesis, after significant simplifications, submitted to the Indian Institute of Science in 2014.
} 
(cf. [17]), symmetrized bi-disc (cf. [3]) and the annulus algebras (cf. [2]). Counter examples are known for domains of connectivity $\geq 2$ (cf. [8]) and any ball in $\mathbb{C}^{m}, m \geq 3$, as we will explain below.

Neither Ando's proof of the existence of a unitary dilation for a pair of commuting contractions, nor the counter example to such an existence theorem due to Parrott involved the notion of complete contractivity directly. In the papers [10, 11, 12, it was shown that the examples of Parrott are not even 2 - contractive. In these papers, for any bounded, connected and open set $\Omega \subset \mathbb{C}^{m}$, the homomorphism $\rho_{\mathbf{V}}: \mathcal{O}(\Omega) \rightarrow \mathcal{M}_{p+q}$, induced by an $m$-tuple of $p \times q$ matrices $\mathbf{V}=$ $\left(V_{1}, \ldots, V_{m}\right)$, modeled after the examples of Parrott, was introduced. This was further studied, in depth, by V. Paulsen [18, where he showed that the question of "contractive vs completely contractive" for Parrott like homomorphisms $\rho_{\mathbf{V}}$ is equivalent to the question of "contractive vs completely contractive" for the linear maps $L_{\mathbf{V}}$ from some finite dimensional Banach space $X$ to $\mathcal{M}_{n}(\mathbb{C})$. The existence of linear maps of the form $L_{\mathbf{V}}$ which are contractive but not completely contractive for $m \geq 5$ were found by him. A refinement (see remark at the bottom of p. 76 in [16]) includes the case $m=3,4$, leaving the question of what happens when $m=2$ open. This is Problem 1 on page 79 of [16] in the list of "Open Problems".

For the normed linear space $\left(\mathbb{C}^{2},\|\cdot\|_{\mathbf{A}}\right)$, we show, except when the pair $A_{1}, A_{2}$ is simultaneously diagonalizable, that there is a contractive linear map on $\left(\mathbb{C}^{2},\|\cdot\|_{\mathbf{A}}\right)$ taking values in $p \times q$ matrices, which is not completely contractive.

We point out that the results of Paulsen used deep ideas from geometry of finite dimensional Banach spaces. In contrast, our results are elementary in nature, although the computations, at times, are somewhat involved.

\section{Preliminaries}

The norm $\|\mathbf{z}\|_{\mathbf{A}}=\left\|z_{1} A_{1}+\cdots+z_{m} A_{m}\right\|_{\mathrm{op}}, \mathbf{z} \in \mathbb{C}^{m}$, is obtained from the embedding of the linear space $\mathbb{C}^{m}$ into the $C^{*}$ algebra of $n \times n$ matrices via the map $P_{\mathbf{A}}(z):=z_{1} A_{1}+\cdots+z_{m} A_{m}$. Let $\Omega_{\mathbf{A}} \subset \mathbb{C}^{m}$ be the unit ball with respect to the norm $\|\cdot\|_{\mathbf{A}}$. Let $\mathcal{O}\left(\Omega_{\mathbf{A}}\right)$ denote the algebra of functions each of which is holomorphic on some open set containing the closed unit ball $\bar{\Omega}_{\mathbf{A}}$. Given $p \times q$ matrices $V_{1}, \ldots, V_{m}$ and a function $f \in \mathcal{O}\left(\Omega_{\mathbf{A}}\right)$, define

$$
\rho_{\mathbf{V}}(f):=\left(\begin{array}{cc}
f(w) I_{p} & \sum_{i=1}^{m} \partial_{i} f(w) V_{i} \\
0 & f(w) I_{q}
\end{array}\right) \text { for a fixed } w \in \Omega_{\mathbf{A}} .
$$

Clearly, $\rho_{\mathbf{V}}:\left(\mathcal{O}\left(\Omega_{\mathbf{A}}\right),\|\cdot\|_{\infty}\right) \rightarrow\left(\mathcal{M}_{p+q}(\mathbb{C}),\|\cdot\|_{\text {op }}\right)$ defines an algebra homomorphism.

At the outset we point out the interesting and useful fact that $\rho_{\mathbf{V}}$ is contractive on $\mathcal{O}\left(\Omega_{\mathbf{A}}\right)$ if and only if it is contractive on the subset of functions which vanish at $w$. This is the content of the following lemma. The proof is reproduced from [18, Lemma 5.1], a direct proof appears in [10, Lemma 3.3].

Lemma 2.1. $\sup _{\|f\|_{\infty}=1}\left\{\left\|\rho_{\mathbf{V}}(f)\right\|_{\text {op }}: f \in \mathcal{O}\left(\Omega_{\mathbf{A}}\right)\right\} \leq 1$ if and only if $\sup _{\|g\|_{\infty}=1}\left\{\left\|\rho_{\mathbf{V}}(g)\right\|_{\text {op }}: g \in\right.$ $\left.\mathcal{O}\left(\Omega_{\mathbf{A}}\right), g(w)=0\right\} \leq 1$.

Proof. The implication in one direction is obvious. To prove the converse, assume that $\left\|\rho_{\mathbf{V}}(g)\right\| \leq$ 1 for every $g$ such that $g(w)=0$ and $\|g\|_{\infty}=1$.

For $f \in \mathcal{O}\left(\Omega_{\mathbf{A}}\right)$ with $\|f\|_{\infty}=1$ let $\phi_{f(w)}$ be the Möbius map of the disc which maps $f(w)$ to 0 . We let $g=\phi_{f(w)} \circ f$. Then $g(w)=0,\|g\|_{\infty}=1$ and, from our assumption, $\left\|\rho_{\mathbf{V}}(g)\right\| \leq 1$. So

$$
\begin{aligned}
\left\|\rho_{\mathbf{V}}(f)\right\| & =\| \rho_{\mathbf{V}}\left(\phi_{f(w)}^{-1} \circ g \|\right. \\
& =\left\|\phi_{f(w)}^{-1}\left(\rho_{\mathbf{V}}(g)\right)\right\| \text { since } \rho_{\mathbf{V}} \text { is a homomorphism } \\
& \leq 1
\end{aligned}
$$


In the last step we use the von Neumann inequality since $\phi_{f(w)}^{-1}$ is a rational function from the disc to itself.

Note: For the rest of this work, we restrict to the case where $w=0$ in the definition (2.1) of $\rho_{\mathbf{V}}$ above.

The following lemma provides a characterization of the unit ball $\Omega_{\mathbf{A}}^{*}$ with respect to the dual norm $\|\cdot\|_{\mathbf{A}}^{*}$ in $\mathbb{C}^{m}$, that is $\Omega_{\mathbf{A}}^{*}=\left(\mathbb{C}^{m},\|\cdot\|_{\mathbf{A}}^{*}\right)_{1}$.

Lemma 2.2. The dual unit ball

$$
\Omega_{\mathbf{A}}^{*}=\left\{\left(\partial_{1} f(0), \partial_{2} f(0), \cdots, \partial_{m} f(0)\right): f \in \operatorname{Hol}\left(\Omega_{\mathbf{A}}, \mathbb{D}\right), f(0)=0\right\} .
$$

Proof. Given $\mathbf{z} \in \mathbb{C}^{m}$ such that $\|\mathbf{z}\|_{\mathbf{A}}=1$ and $f \in \operatorname{Hol}\left(\Omega_{\mathbf{A}}, \mathbb{D}\right), f(0)=0$, we define $g_{\mathbf{z}}: \mathbb{D} \rightarrow \Omega_{\mathbf{A}}$ by

$$
g_{\mathbf{z}}(\lambda)=\lambda \mathbf{z}, \lambda \in \mathbb{D} .
$$

Then $f \circ g_{\mathbf{z}}: \mathbb{D} \rightarrow \mathbb{D}$ with $\left(f \circ g_{\mathbf{z}}\right)(0)=0$. Applying the Schwarz Lemma to the function $\left(f \circ g_{\mathbf{z}}\right)$ we get

$$
1 \geq\left|\left(f \circ g_{\mathbf{z}}\right)^{\prime}(0)\right|=\left|f^{\prime}\left(g_{\mathbf{z}}(0)\right) \cdot g_{\mathbf{z}}^{\prime}(0)\right|=\left|f^{\prime}(0) \cdot g_{\mathbf{z}}^{\prime}(0)\right|=\left|f^{\prime}(0) \cdot \mathbf{z}\right| .
$$

In the above, $f^{\prime}(0) \cdot \mathbf{z}=\sum_{i=1}^{m}\left(\partial_{i} f(0)\right) z_{i}$, etc.

Hence $\left(\partial_{1} f(0), \partial_{2} f(0), \cdots, \partial_{m} f(0)\right) \in \Omega_{\mathbf{A}}^{*}$.

Conversely, given $\mathbf{w} \in \Omega_{\mathbf{A}}^{*}$, we define $f_{\mathbf{w}}(\mathbf{z})=\mathbf{w} \cdot \mathbf{z}$ so that $\partial_{i} f_{\mathbf{w}}(0)=w_{i}$.

2.1. The Maps $L_{\mathbf{V}}^{(k)}$ : From Lemma 2.1 above it follows that

$$
\left\|\rho_{\mathbf{V}}\right\| \leq 1 \text { if and only if } \sup _{\|f\|_{\infty}=1, f(0)=0}\left\|\sum_{i=1}^{m} \partial_{i} f(0) V_{i}\right\|_{\text {op }} \leq 1 .
$$

Considering Lemma 2.2 and the equivalence (2.2) above it is natural to consider the induced linear $\operatorname{map} L_{\mathbf{V}}:\left(\mathbb{C}^{m},\|\cdot\|_{\mathbf{A}}^{*}\right) \rightarrow \mathcal{M}_{p, q}(\mathbb{C})$ given by

$$
L_{\mathbf{V}}(w)=w_{1} V_{1}+\cdots+w_{m} V_{m} .
$$

It follows from (2.2) above that

$$
\left\|\rho_{\mathbf{V}}\right\| \leq 1 \text { if and only if }\left\|L_{\mathbf{V}}\right\| \leq 1 .
$$

We will show now that the complete contractivity of $\rho_{\mathbf{V}}$ and $L_{\mathbf{V}}$ are also related similarly.

For a holomorphic function $F: \Omega_{\mathbf{A}} \rightarrow \mathcal{M}_{k}$ with $\|F\|=\sup _{\mathbf{z} \in \boldsymbol{\Omega}_{\mathbf{A}}}\|F(\mathbf{z})\|$, we define

$$
\rho_{\mathbf{V}}^{(k)}(F):=\left(\rho_{\mathbf{V}}\left(F_{i j}\right)\right)_{i, j=1}^{m}=\left(\begin{array}{cc}
F(0) \otimes I & \sum_{i=1}^{m}\left(\partial_{i} F(0)\right) \otimes V_{i} \\
0 & F(0) \otimes I
\end{array}\right) .
$$

Using a method similar to that used for $\rho_{\mathbf{V}}$ it can be shown that

$$
\left\|\rho_{\mathbf{V}}^{(k)}\right\| \leq 1 \text { if and only if } \sup _{F}\left\{\left\|\sum_{i=1}^{m}\left(\partial_{i} F(0)\right) \otimes V_{i}\right\|: F \in \operatorname{Hol}\left(\Omega_{\mathbf{A}},\left(\mathcal{M}_{k}\right)_{1}\right), F(0)=0\right\} \leq 1,
$$

that is, (by repeating the argument used for $\rho_{\mathbf{V}}$ ) we have

$$
\left\|\rho_{\mathbf{V}}^{(k)}\right\| \leq 1 \text { if and only if }\left\|L_{\mathbf{V}}^{(k)}\right\| \leq 1,
$$

where

is the map

$$
L_{\mathbf{V}}^{(k)}:\left(\mathbb{C}^{m} \otimes \mathcal{M}_{k},\|\cdot\|_{\mathbf{A}, k}^{*}\right) \rightarrow\left(\mathcal{M}_{k} \otimes \mathcal{M}_{p, q},\|\cdot\|_{\mathrm{op}}\right)
$$

$$
L_{\mathbf{V}}^{(k)}\left(\Theta_{1}, \Theta_{2}, \cdots, \Theta_{m}\right)=\Theta_{1} \otimes V_{1}+\Theta_{2} \otimes V_{2}+\cdots+\Theta_{m} \otimes V_{m} \text { for } \Theta_{1}, \Theta_{2}, \cdots, \Theta_{m} \in \mathcal{M}_{k}
$$


2.2. The polynomial $P_{\mathbf{A}}$. A very useful construct for our analysis is the matrix valued polynomial $P_{\mathbf{A}}$ with $P_{\mathbf{A}}\left(\Omega_{\mathbf{A}}\right) \subseteq\left(\mathcal{M}_{n},\|\cdot\|_{\text {op }}\right)_{1}$ defined by

$$
P_{\mathbf{A}}\left(z_{1}, z_{2}, \cdots, z_{m}\right)=z_{1} A_{1}+z_{2} A_{2}+\cdots+z_{m} A_{m},
$$

with the norm $\left\|P_{\mathbf{A}}\right\|_{\infty}=\sup _{\left(z_{1}, \cdots, z_{m}\right) \in \Omega_{\mathbf{A}}}\left\|P_{\mathbf{A}}\left(z_{1}, \cdots, z_{m}\right)\right\|_{\text {op }}$. Note that $\left\|P_{\mathbf{A}}\right\|_{\infty}=1$ by definition. The typical procedure used to show the existence of a homomorphism which is contractive but not completely contractive is to construct a contractive homomorphism $\rho_{\mathbf{V}}$ (by a suitable choice of $\mathbf{V}$ ) and to then show that its evaluation on $P_{\mathbf{A}}$, that is, $\rho_{\mathbf{V}}^{(n)}\left(P_{\mathbf{A}}\right)$, has norm greater than 1 .

2.3. Homomorphisms induced by $m$-vectors. We now consider the special situation when the matrices $V_{1}, \cdots, V_{m}$ are vectors in $\mathbb{C}^{m}$ realized as row $m$-vectors. For $w=\left(w_{1}, \ldots, w_{m}\right)$ in some bounded domain $\Omega \subseteq \mathbb{C}^{m}$, the commuting $m$-tuple of $(m+1) \times(m+1)$ matrices of the form $\left(\begin{array}{cc}w_{i} & V_{i} \\ 0 & w_{i} I_{m}\end{array}\right), 1 \leq i \leq m$, induce the homomorphism $\rho_{\boldsymbol{V}}$ via the usual functional calculus, that is,

$$
\rho_{\boldsymbol{V}}(f):=f\left(\left(\begin{array}{cc}
w_{1} & V_{1} \\
0 & w_{1} I_{m}
\end{array}\right), \ldots,\left(\begin{array}{cc}
w_{m} & V_{m} \\
0 & w_{m} I_{m}
\end{array}\right)\right), f \in \mathcal{O}(\Omega),
$$

see (2.1). The localization of a commuting $m$ - tuple $\boldsymbol{T}$ of operators in the class $B_{1}(\Omega)$, introduced in $([\underline{5},[6])$, is also a commuting $m$ - tuple of $(m+1) \times(m+1)$ matrices, which is exactly of the form described above. The vectors $V_{1}, \ldots, V_{m}$ appearing in such localizations are given explicitly in terms of the curvature of the holomorphic Hermitian vector bundle corresponding to $\boldsymbol{T}$ as shown in [6]. The contractivity of the homomorphism $\rho_{\boldsymbol{V}}$ then results in curvature inequalities (see [9, 11, 12, 13]).

Let $V_{i}=\left(\begin{array}{llll}v_{i 1} & v_{i 2} & \cdots & v_{i m}\end{array}\right), i=1, \cdots, m$. The propositions below are useful to study contractivity and complete contractivity in this special case, where, as before, we assume that $\Omega=\Omega_{\boldsymbol{A}}$ and $w=0$.

Proposition 2.3. The following are equivalent:

(i) $\rho_{\mathbf{V}}$ is contractive,

(ii) $\sup _{\sum_{j=1}^{m}\left|z_{j}\right|^{2} \leq 1}\left\|\sum_{j=1}^{m} z_{j} B_{j}\right\|_{\mathrm{op}}^{2} \leq 1$, where $B_{j}=\sum_{i=1}^{m} v_{i j} A_{i}$.

Proof. We have shown that the homomorphisms $\left\|\rho_{\mathbf{V}}\right\|_{\mathcal{O}\left(\Omega_{\mathbf{A}}\right) \rightarrow \mathcal{M}_{m+1}(\mathbb{C})}$ is contractive if and only if the linear map $\left\|L_{\mathbf{V}}\right\|_{\left(\mathbb{C}^{m},\|\cdot\|_{\mathbf{A}}^{*}\right) \rightarrow\left(\mathbb{C}^{m},\|\cdot\|_{2}\right)}$ is contractive (equivalently if $\left\|L_{\mathbf{V}}^{*}\right\|_{\left(\mathbb{C}^{m},\|\cdot\|_{2}\right) \rightarrow\left(\mathbb{C}^{m},\|\cdot\|_{\mathbf{A}}\right)}$ is contractive).

The matrix representation of $L_{\mathbf{V}}^{*}$ is $\left(\begin{array}{ccc}v_{11} & \cdots & v_{1 m} \\ \vdots & \ddots & \vdots \\ v_{m 1} & \cdots & v_{m m}\end{array}\right)$.

Hence the contractivity of $L_{\mathbf{V}}^{*}$ is given by the condition that

$$
\sup _{\sum_{j=1}^{m}\left|z_{j}\right|^{2} \leq 1}\left\|\left(\begin{array}{ccc}
v_{11} & \cdots & v_{1 m} \\
\vdots & \ddots & \vdots \\
v_{m 1} & \cdots & v_{m m}
\end{array}\right)\left(\begin{array}{c}
z_{1} \\
\vdots \\
z_{m}
\end{array}\right)\right\|_{\mathbf{A}} \leq 1
$$

From the definition of $\|\cdot\|_{\mathbf{A}}$ it follows that

$$
\left\|L_{\mathbf{V}}^{*}\right\|_{\left(\mathbb{C}^{m},\|\cdot\|_{2}\right) \rightarrow\left(\mathbb{C}^{m},\|\cdot\|_{\mathbf{A}}\right)} \leq 1 \text { if and only if } \sup _{\sum_{j=1}^{m}\left|z_{j}\right|^{2} \leq 1}\left\|\sum_{j=1}^{m} z_{j} B_{j}\right\|_{\text {op }}^{2} \leq 1
$$

where $B_{j}=\sum_{i=1}^{m} v_{i j} A_{i}$.

In particular, if $V_{1}=\left(\begin{array}{ll}u & 0\end{array}\right)$ and $V_{2}=\left(\begin{array}{ll}0 & v\end{array}\right)$, the condition (ii) above becomes

$$
\sup _{\sum_{j=1}^{2}\left|z_{j}\right|^{2} \leq 1}\left\|z_{1} u A_{1}+z_{2} v A_{2}\right\|^{2} \leq 1,
$$

which is equivalent to the following two conditions: 
(i) $|u|^{2} \leq \frac{1}{\left\|A_{1}^{*}\right\|^{2}}$ or $|v|^{2} \leq \frac{1}{\left\|A_{2}^{*}\right\|^{2}}$

(ii)

$$
\inf _{\beta \in \mathbb{C}^{2},\|\beta\|=1}\left\{1-|u|^{2}\left\|A_{1}^{*} \beta\right\|^{2}-|v|^{2}\left\|A_{2}^{*} \beta\right\|^{2}+|u v|^{2}\left(\left\|A_{1}^{*} \beta\right\|^{2}\left\|A_{2}^{*} \beta\right\|^{2}-\left|\left\langle A_{1} A_{2}^{*} \beta, \beta\right\rangle\right|^{2}\right)\right\} \geq 0 .
$$

Proposition 2.4. The following are equivalent:

(i) $\left\|\rho_{\mathbf{V}}^{(n)}\left(P_{\mathbf{A}}\right)\right\| \leq 1$,

(ii) The $n \times m n$ matrix $\left(\begin{array}{llll}B_{1} & B_{2} & \cdots & B_{m}\end{array}\right)$ is contractive, where $B_{j}=\sum_{i=1}^{m} v_{i j} A_{i}$.

Proof. Since $P_{\mathbf{A}}(0)=0$, it follows from the definition (2.3) that $\left\|\rho_{\mathbf{V}}^{(n)}\left(P_{\mathbf{A}}\right)\right\| \leq 1$ if and only if

$$
\left\|A_{1} \otimes V_{1}+\ldots+A_{m} \otimes V_{m}\right\| \leq 1 .
$$

For $V_{i}=\left(\begin{array}{lll}v_{i 1} & \cdots & v_{i m}\end{array}\right)$, we have

$$
A_{1} \otimes V_{1}+\ldots+A_{m} \otimes V_{m}=\left(\begin{array}{llll}
B_{1} & B_{2} & \cdots & B_{m}
\end{array}\right)
$$

Thus $\left\|\rho_{V}^{(n)}\left(P_{\mathbf{A}}\right)\right\| \leq 1$ if and only if $\left\|\left(\begin{array}{llll}B_{1} & B_{2} & \cdots & B_{m}\end{array}\right)\right\| \leq 1$.

In particular if $V_{1}=\left(\begin{array}{ll}u & 0\end{array}\right)$ and $V_{2}=\left(\begin{array}{ll}0 & v\end{array}\right)$ the condition (ii) above becomes

$$
\inf _{\beta \in \mathbb{C}^{2},\|\beta\|=1}\left\{1-|u|^{2}\left\|A_{1}^{*} \beta\right\|^{2}-|v|^{2}\left\|A_{2}^{*} \beta\right\|^{2}\right\} \geq 0 .
$$

Note: For most of this paper we will restrict to the two dimensional case. That is, we consider $\mathbb{C}^{2}$ with the norm defined by a matrix pair $\left(A_{1}, A_{2}\right)$. In fact, for the most part, we even restrict to the situation where $A_{1}, A_{2}$ are $2 \times 2$ matrices. This is adequate for our primary purpose of constructing homomorphisms of $\mathcal{O}\left(\Omega_{\mathbf{A}}\right)$ which are contractive but not completely contractive. Many of the results can be adapted to higher dimensional situation.

\section{Defining Function and Test Functions}

Recall the matrix valued polynomial $P_{\mathbf{A}}: \Omega_{\mathbf{A}} \rightarrow\left(\mathcal{M}_{2},\|\cdot\|_{\text {op }}\right)_{1}$ defined earlier by

$$
P_{\mathbf{A}}\left(z_{1}, z_{2}\right)=z_{1} A_{1}+z_{2} A_{2},
$$

where $\left(\mathcal{M}_{2},\|\cdot\|_{\text {op }}\right)_{1}$ is the matrix unit ball with respect to the operator norm. For $\left(z_{1}, z_{2}\right)$ in $\Omega_{\mathbf{A}}$, the norm

$$
\left\|P_{\mathbf{A}}\right\|_{\infty}:=\sup _{\left(z_{1}, z_{2}\right) \in \Omega_{\mathbf{A}}}\left\|P_{\mathbf{A}}\left(z_{1}, z_{2}\right)\right\|_{\text {op }}=1
$$

by definition of the polynomial $P_{\mathbf{A}}$.

Let $\mathbb{B}^{2}$ be the unit ball in $\mathbb{C}^{2}$. For $(\alpha, \beta) \in \mathbb{B}^{2} \times \mathbb{B}^{2}$, define $p_{\mathbf{A}}^{(\alpha, \beta)}: \Omega_{\mathbf{A}} \rightarrow \mathbb{D}$ to be the linear map

$$
p_{\mathbf{A}}^{(\alpha, \beta)}\left(z_{1}, z_{2}\right)=\left\langle P_{\mathbf{A}}\left(z_{1}, z_{2}\right) \alpha, \beta\right\rangle=z_{1}\left\langle A_{1} \alpha, \beta\right\rangle+z_{2}\left\langle A_{2} \alpha, \beta\right\rangle .
$$

The sup norm $\left\|p_{\mathbf{A}}^{(\alpha, \beta)}\right\|_{\infty}$, for any pair of vectors $(\alpha, \beta)$ in $\mathbb{B}^{2} \times \mathbb{B}^{2}$, is at most 1 by definition. Let $\mathcal{P}_{\mathbf{A}}$ denote the collection of linear functions $\left\{p_{\mathbf{A}}^{(\alpha, \beta)}:(\alpha, \beta) \in \mathbb{B}^{2} \times \mathbb{B}^{2}\right\}$.

The map $P_{\mathbf{A}}$ which we call the 'Defining Function' of the domain and the collection of functions $\mathcal{P}_{\mathbf{A}}$ which we call a family of 'Test Functions' encode a significant amount of information relevant to our purpose about the homomorphism $\rho_{\mathbf{V}}$. For instance $\rho_{\mathbf{V}}$ is contractive if its restriction to $\mathcal{P}_{\mathbf{A}}$ is contractive. Also the lack of complete contractivity can often be shown by evaluating $\rho_{\mathbf{V}}^{(2)}$ on $P_{\mathbf{A}}$. Some of the details are outlined in the lemma below. 
Lemma 3.1. In the notation fixed in the preceding discussion, we have

(i) $\sup _{\|\alpha\|=\|\beta\|=1}\left\|\rho_{\mathbf{V}}\left(p_{\mathbf{A}}^{(\alpha, \beta)}\right)\right\| \leq\left\|\rho_{\mathbf{V}}^{(2)}\left(P_{\mathbf{A}}\right)\right\|$,

(ii) $\rho_{\mathbf{V}}$ is contractive if and only if $\sup _{\|\alpha\|=\|\beta\|=1}\left\|\rho_{\mathbf{V}}\left(p_{\mathbf{A}}^{(\alpha, \beta)}\right)\right\| \leq 1$.

Proof of (i). Since

$$
\rho_{\mathbf{V}}\left(p_{\mathbf{A}}^{(\alpha, \beta)}\right)=\left(\begin{array}{cc}
0 & \left(\partial_{1} p_{\mathbf{A}}^{(\alpha, \beta)}(0)\right) V_{1}+\left(\partial_{2} p_{\mathbf{A}}^{(\alpha, \beta)}(0)\right) V_{2} \\
0 & 0
\end{array}\right)
$$

by definition, it follows that

$$
\begin{aligned}
\left\|\rho_{\mathbf{V}}\left(p_{\mathbf{A}}^{(\alpha, \beta)}\right)\right\| & =\left\|\left(\partial_{1} p_{\mathbf{A}}^{(\alpha, \beta)}(0)\right) V_{1}+\left(\partial_{2} p_{\mathbf{A}}^{(\alpha, \beta)}(0)\right) V_{2}\right\|_{\text {op }} \\
& =\left\|\left\langle A_{1} \alpha, \beta\right\rangle V_{1}+\left\langle A_{2} \alpha, \beta\right\rangle V_{2}\right\|_{\text {op }} \\
& =\sup _{\|u\|=\|v\|=1}\left|\left\langle A_{1} \alpha, \beta\right\rangle\left\langle V_{1} u, v\right\rangle+\left\langle A_{2} \alpha, \beta\right\rangle\left\langle V_{2} u, v\right\rangle\right| .
\end{aligned}
$$

Hence

$$
\begin{aligned}
\sup _{\|\alpha\|=\|\beta\|=1}\left\|\rho_{\mathbf{V}}\left(p_{\mathbf{A}}^{(\alpha, \beta)}\right)\right\| & =\sup _{\|\alpha\|=\|\beta\|=1} \sup _{\|u\|=\|v\|=1}\left|\left\langle A_{1} \alpha, \beta\right\rangle\left\langle V_{1} u, v\right\rangle+\left\langle A_{2} \alpha, \beta\right\rangle\left\langle V_{2} u, v\right\rangle\right| \\
& =\sup _{\|\alpha\|=\|\beta\|=1} \sup _{\|u\|=\|v\|=1}\left|\left\langle\left(A_{1} \otimes V_{1}+A_{2} \otimes V_{2}\right) \alpha \otimes u, \beta \otimes v\right\rangle\right| \\
& =\sup _{\|\alpha\|=\|\beta\|=1} \sup _{\|u\|=\|v\|=1}\left|\left\langle\rho_{\mathbf{V}}^{(2)}\left(P_{\mathbf{A}}\right) \alpha \otimes u, \beta \otimes v\right\rangle\right| \\
& \leq\left\|\rho_{\mathbf{V}}^{(2)}\left(P_{\mathbf{A}}\right)\right\| .
\end{aligned}
$$

Proof of (ii). As indicated earlier the contractivity of $\rho_{\mathbf{V}}$ is equivalent to the contractivity of

$$
L_{\mathbf{V}}:\left(\mathbb{C}^{2},\|\cdot\|_{\mathbf{A}}^{*}\right) \rightarrow\left(\mathcal{M}_{p, q},\|\cdot\|_{\mathrm{op}}\right)
$$

given by the formula

$$
L_{\mathbf{V}}\left(\omega_{1}, \omega_{2}\right)=\omega_{1} V_{1}+\omega_{2} V_{2}
$$

So we identify the conditions for the contractivity of $L_{\mathbf{V}}$ :

$$
\begin{aligned}
\left\|L_{\mathbf{V}}\right\| & =\sup _{\left\|\left(\omega_{1}, \omega_{2}\right)\right\|_{\mathbf{A}}^{*} \leq 1}\left\|\omega_{1} V_{1}+\omega_{2} V_{2}\right\|_{\text {op }} \\
& =\sup _{\left\|\left(\omega_{1}, \omega_{2}\right)\right\|_{\mathbf{A}}^{*} \leq 1} \sup _{\|u\|=\|v\|=1}\left|\omega_{1}\left\langle V_{1} u, v\right\rangle+\omega_{2}\left\langle V_{2} u, v\right\rangle\right| .
\end{aligned}
$$

Hence, since $\left(\omega_{1}, \omega_{2}\right)$ lies in the dual of $\Omega_{\mathbf{A}}$,

$$
\begin{aligned}
\left\|L_{\mathbf{V}}\right\| \leq 1 & \Longleftrightarrow\left(\left\langle V_{1} u, v\right\rangle,\left\langle V_{2} u, v\right\rangle\right) \in \Omega_{\mathbf{A}} \forall u, v \text { such that }\|u\|=\|v\|=1 \\
& \Longleftrightarrow \sup _{\|u\|=\|v\|=1}\left\|\left\langle V_{1} u, v\right\rangle A_{1}+\left\langle V_{2} u, v\right\rangle A_{2}\right\|_{\text {op }} \leq 1 \\
& \Longleftrightarrow \sup _{\|\alpha\|=\|\beta\|=1} \sup _{\|u\|=\|v\|=1}\left|\left\langle A_{1} \alpha, \beta\right\rangle\left\langle V_{1} u, v\right\rangle+\left\langle A_{2} \alpha, \beta\right\rangle\left\langle V_{2} u, v\right\rangle\right| \leq 1 \\
& \Longleftrightarrow \sup _{\|\alpha\|=\|\beta\|=1}\left\|\rho_{\mathbf{V}}\left(p_{\mathbf{A}}^{(\alpha, \beta)}\right)\right\| \leq 1 \text { from (3.1) above. }
\end{aligned}
$$

As mentioned earlier, by choosing a pair $\left(V_{1}, V_{2}\right)$ such that the inequality in $(i)$ above is strict, we can often construct a contractive homomorphism which is not completely contractive. We illustrate below choices of $\left(V_{1}, V_{2}\right)$ for the Euclidean ball for which the inequality is strict. 
Example 3.2. (Euclidean Ball) Choosing $\mathbf{A}=\left(\left(\begin{array}{ll}1 & 0 \\ 0 & 0\end{array}\right),\left(\begin{array}{ll}0 & 1 \\ 0 & 0\end{array}\right)\right)$, we see that $\Omega_{\mathbf{A}}$ defines the Euclidean ball $\mathbb{B}^{2}$ in $\mathbb{C}^{2}$. Choose $V_{1}=\left(v_{11} v_{12}\right), V_{2}=\left(v_{21} v_{22}\right)$. We will prove that

$$
\sup _{\|\alpha\|=\|\beta\|=1}\left\|\rho_{\mathbf{V}}\left(p_{\mathbf{A}}^{(\alpha, \beta)}\right)\right\|<\left\|\rho_{\mathbf{V}}^{(2)}\left(P_{\mathbf{A}}\right)\right\|_{\mathrm{op}},
$$

if $V_{1}$ and $V_{2}$ are linearly independent.

In fact we can choose $\left(V_{1}, V_{2}\right)$ such that $\sup _{\|\alpha\|=\|\beta\|=1}\left\|\rho_{\mathbf{V}}\left(p_{\mathbf{A}}^{(\alpha, \beta)}\right)\right\| \leq 1$ and $\left\|\rho_{\mathbf{V}}^{(2)}\left(P_{\mathbf{A}}\right)\right\|_{\text {op }}>$ 1. This example of a contractive homomorphism of the ball algebra which is not completely contractive was found in [10, 11].

Theorem 3.3. For $\Omega_{\mathbf{A}}=\mathbb{B}^{2}$, let $V_{1}=\left(\begin{array}{ll}v_{11} & v_{12}\end{array}\right), V_{2}=\left(\begin{array}{ll}v_{21} & v_{22}\end{array}\right)$. Then

(i) $\sup _{\|\alpha\|=\|\beta\|=1}\left\|\rho_{\mathbf{V}}\left(p_{\mathbf{A}}^{(\alpha, \beta)}\right)\right\|^{2}=\left\|\left(\begin{array}{ll}v_{11} & v_{12} \\ v_{21} & v_{22}\end{array}\right)\right\|_{\mathrm{op}}^{2}$

(ii) $\left\|\rho_{\mathbf{V}}^{(2)}\left(P_{\mathbf{A}}\right)\right\|_{\mathrm{op}}^{2}=\left\|\left(\begin{array}{ll}v_{11} & v_{12} \\ v_{21} & v_{22}\end{array}\right)\right\|_{\mathrm{HS}}^{2}$ (HS represents the Hilbert - Schmidt norm)

Consequently, $\sup _{\|\alpha\|=\|\beta\|=1}\left\|\rho_{\mathbf{V}}\left(p_{\mathbf{A}}^{(\alpha, \beta)}\right)\right\|<\left\|\rho_{\mathbf{V}}^{(2)}\left(P_{\mathbf{A}}\right)\right\|_{\text {op }}$ if $V_{1}$ and $V_{2}$ are linearly independent.

Proof. By the definition of $\rho_{\mathbf{V}}$ we have

$$
\begin{aligned}
\sup _{\|\alpha\|=\|\beta\|=1}\left\|\rho_{\mathbf{V}}\left(p_{\mathbf{A}}^{(\alpha, \beta)}\right)\right\|^{2} & =\sup _{\|\alpha\|=\|\beta\|=\|u\|=\|v\|=1}\left|\left\langle A_{1} \alpha, \beta\right\rangle\left\langle V_{1} u, v\right\rangle+\left\langle A_{2} \alpha, \beta\right\rangle\left\langle V_{2} u, v\right\rangle\right|^{2} \\
& =\sup _{\|\alpha\|=\|\beta\|=\|u\|=1}\left|\alpha_{1}\left(v_{11} u_{1}+v_{12} u_{2}\right)+\alpha_{2}\left(v_{21} u_{1}+v_{22} u_{2}\right)\right|^{2}\left|\beta_{1}\right|^{2} \\
& =\sup _{\|\alpha\|=\|u\|=1}\left|\alpha_{1}\left(v_{11} u_{1}+v_{12} u_{2}\right)+\alpha_{2}\left(v_{21} u_{1}+v_{22} u_{2}\right)\right|^{2} \\
& =\sup _{\|u\|=1}\left|v_{11} u_{1}+v_{12} u_{2}\right|^{2}+\left|v_{21} u_{1}+v_{22} u_{2}\right|^{2} \\
& =\left\|\left(\begin{array}{ll}
v_{11} & v_{12} \\
v_{21} & v_{22}
\end{array}\right)\right\|_{\text {op }}^{2} .
\end{aligned}
$$

On the other hand, we have

$$
\left\|\rho_{\mathbf{V}}^{(2)}\left(P_{\mathbf{A}}\right)\right\|_{\mathrm{op}}^{2}=\left\|V_{1}\right\|^{2}+\left\|V_{2}\right\|^{2}=\left\|\left(\begin{array}{cc}
v_{11} & v_{12} \\
v_{21} & v_{22}
\end{array}\right)\right\|_{\mathrm{HS}}^{2} .
$$

If $V_{1}$ and $V_{2}$ are linearly independent

$$
\left\|\left(\begin{array}{ll}
v_{11} & v_{12} \\
v_{21} & v_{22}
\end{array}\right)\right\|_{\mathrm{op}}^{2}<\left\|\left(\begin{array}{ll}
v_{11} & v_{12} \\
v_{21} & v_{22}
\end{array}\right)\right\|_{\mathrm{HS}}^{2}
$$

and we have

$$
\sup _{\|\alpha\|=\|\beta\|=1}\left\|\rho_{\mathbf{V}}\left(p_{\mathbf{A}}^{(\alpha, \beta)}\right)\right\|<\left\|\rho_{\mathbf{V}}^{(2)}\left(P_{\mathbf{A}}\right)\right\|_{\mathrm{op}}
$$

Now choose $V_{1}=\left(\begin{array}{ll}1 & 0\end{array}\right)$ and $V_{2}=\left(\begin{array}{ll}0 & 1\end{array}\right)$. From Lemma 3.1 and Theorem 3.3 it follows that $\rho_{\mathbf{V}}$ is contractive but $\left\|\rho_{\mathbf{V}}^{(2)}\left(P_{\mathbf{A}}\right)\right\|=\sqrt{2}$.

\section{Unitary Equivalence and Linear Equivalence}

If $U$ and $W$ are $2 \times 2$ unitary matrices and $\widetilde{\mathbf{A}}=\left(U A_{1} W, U A_{2} W\right)$, then

$$
\left\|\left(z_{1}, z_{2}\right)\right\|_{\mathbf{A}}=\left\|z_{1} A_{1}+z_{2} A_{2}\right\|_{\mathrm{op}}=\left\|z_{1}\left(U A_{1} W\right)+z_{2}\left(U A_{2} W\right)\right\|_{\mathrm{op}}=\left\|\left(z_{1}, z_{2}\right)\right\|_{\widetilde{\mathbf{A}}} .
$$

There are, therefore, various choices of the matrix pair $\left(A_{1}, A_{2}\right)$ related as above which give rise to the same norm. We use this freedom to ensure that $A_{1}$ is diagonal. Consider the invertible linear transformation $\left(\tilde{z}_{1}, \tilde{z}_{2}\right) \mapsto\left(z_{1}, z_{2}\right)$ on $\mathbb{C}^{2}$ defined as follows: 
For $\tilde{\mathbf{z}}=\left(\tilde{z}_{1}, \tilde{z}_{2}\right)$ in $\mathbb{C}^{2}$, let

$$
\begin{aligned}
& z_{1}=p \tilde{z}_{1}+q \tilde{z}_{2} \\
& z_{2}=r \tilde{z}_{1}+s \tilde{z}_{2},
\end{aligned}
$$

where $p, q, r, s \in \mathbb{C}$. Then

$$
\left\|\left(z_{1}, z_{2}\right)\right\|_{\mathbf{A}}=\left\|\left(\tilde{z}_{1}, \tilde{z}_{2}\right)\right\|_{\widetilde{\mathbf{A}}},
$$

where $\widetilde{\mathbf{A}}$ is related to $\mathbf{A}$ as follows:

$$
\begin{aligned}
& \widetilde{A}_{1}=p A_{1}+r A_{2} \\
& \widetilde{A}_{2}=q A_{1}+s A_{2} .
\end{aligned}
$$

More concisely, if $T$ is the linear transformation above on $\mathbb{C}^{2}$, then

$$
\|T \tilde{\mathbf{z}}\|_{\mathbf{A}}=\|\tilde{\mathbf{z}}\|_{\mathbf{A}(T \otimes I)} .
$$

In particular $T$ maps $\Omega_{\widetilde{\mathbf{A}}}$ onto $\Omega_{\mathbf{A}}$.

Lemma 4.1. For $k=1,2, \ldots$, the contractivity of the linear maps $L_{\mathbf{V}}^{(k)}$ defined on $\left(\mathbb{C}^{2} \otimes \mathcal{M}_{k},\|\cdot\|_{\widetilde{\mathbf{A}}, k}^{*}\right)$ determine the contractivity of the linear maps $L_{\widetilde{\mathbf{V}}}^{(k)}$ defined on $\left(\mathbb{C}^{2} \otimes \mathcal{M}_{k},\|\cdot\|_{\mathbf{A}, k}^{*}\right)$ and conversely, where $\widetilde{\mathbf{A}}=\mathbf{A}(T \otimes I)$ and $\widetilde{\mathbf{V}}=(T \otimes I) \mathbf{V}$.

Proof. For $k=1,2, \ldots$, we have to show that

$$
\left\|L_{\mathbf{V}}^{(k)}\right\|_{\left(\mathbb{C}^{2} \otimes \mathcal{M}_{k},\|\cdot\|_{\tilde{\mathbf{A}}, k}^{*}\right) \rightarrow\left(\mathcal{M}_{k} \otimes \mathcal{M}_{p, q},\|\cdot\|_{\mathrm{op}}\right)} \leq 1 \Longleftrightarrow\left\|L_{\widetilde{\mathbf{V}}}^{(k)}\right\|_{\left(\mathbb{C}^{2} \otimes \mathcal{M}_{k},\|\cdot\|_{\mathbf{A}, k}^{*}\right) \rightarrow\left(\mathcal{M}_{k} \otimes \mathcal{M}_{p, q},\|\cdot\|_{\mathrm{op}}\right)} \leq 1 .
$$

We prove this result for the case $k=1$, that is, for the map $L_{\mathbf{V}}$. The proof for the general case is similar.

Consider the bijection between the spaces $\left\{f \in \operatorname{Hol}\left(\Omega_{\mathbf{A}}, \mathbb{D}\right), f(0)=0\right\}$ and $\left\{\widetilde{f} \in \operatorname{Hol}\left(\Omega_{\widetilde{\mathbf{A}}}, \mathbb{D}\right), \widetilde{f}(0)=0\right\}$ defined as follows:

$$
f \mapsto \tilde{f}=f \circ T, \tilde{f} \mapsto f=\tilde{f} \circ T^{-1}
$$

Using this bijection

$$
\begin{aligned}
& \left\|L_{\mathbf{V}}\right\|_{\left(\mathbb{C}^{2},\|\cdot\|_{\tilde{\mathbf{A}}}^{*}\right) \rightarrow\left(\mathcal{M}_{p, q},\|\cdot\|_{\mathrm{op}}\right)} \leq 1 \Longleftrightarrow \sup _{\widetilde{f}}\left\{\|D \widetilde{f}(0) \cdot \mathbf{V}\|_{\text {op }}: \widetilde{f} \in \operatorname{Hol}\left(\Omega_{\widetilde{\mathbf{A}}}, \mathbb{D}\right), \widetilde{f}(0)=0\right\} \leq 1 \\
& \Longleftrightarrow \sup _{f}\left\{\|D(f \circ T)(0) \cdot \mathbf{V}\|_{\text {op }}: f \in \operatorname{Hol}\left(\Omega_{\mathbf{A}}, \mathbb{D}\right), f(0)=0\right\} \leq 1 \\
& \Longleftrightarrow \sup _{f}\left\{\|D f(0) T \cdot \mathbf{V}\|_{\text {op }}: f \in \operatorname{Hol}\left(\Omega_{\mathbf{A}}, \mathbb{D}\right), f(0)=0\right\} \leq 1 \\
& \Longleftrightarrow \sup _{f}\left\{\|D f(0) \cdot(T \otimes I) \mathbf{V}\|_{\mathrm{op}}: f \in \operatorname{Hol}\left(\Omega_{\mathbf{A}}, \mathbb{D}\right), f(0)=0\right\} \leq 1 \\
& \Longleftrightarrow\left\|L_{(T \otimes I) \mathbf{V}}\right\|_{\left(\mathbb{C}^{2},\|\cdot\|_{\mathbf{A}}^{*}\right) \rightarrow\left(\mathcal{M}_{p, q},\|\cdot\|_{\text {op }}\right)} \leq 1
\end{aligned}
$$

In the above, $D f$ is a row vector, $T$ is a $2 \times 2$ matrix and by an expression of the form $X \cdot Y$ we mean $\sum_{i=1}^{2} X_{i} Y_{i}$.

It follows that, in our study of the existence of contractive homomorphisms which are not completely contractive, two sets of matrices $\mathbf{A}=\left(A_{1}, A_{2}\right)$ and $\widetilde{\mathbf{A}}=\left(\widetilde{A}_{1}, \widetilde{A}_{2}\right)$ which are related through linear combinations as above yield the same result. We can, therefore, restrict our attention to a subcollection of matrices.

Since $A_{1}$ has already been chosen to be diagonal, we consider transformations as above with $r=0$ to preserve the diagonal structure of $A_{1}$. By choosing the parameters $p, q, s$ suitably we can ensure that one diagonal entry of $A_{1}$ is 1 and the diagonal entries of $A_{2}$ are 1 and 0 . By further 
conjugating with a diagonal unitary and a permutation matrix it follows that we need to consider only the following three families of matrices:

TABLE 1. Cases modulo unitary and linear equivalence

\begin{tabular}{|c|c|}
\hline$A_{1}$ & $A_{2}$ \\
\hline \hline$\left(\begin{array}{ll}1 & 0 \\
0 & d\end{array}\right) d \in \mathbb{C}$ & $\left(\begin{array}{ll}1 & b \\
c & 0\end{array}\right) c \in \mathbb{C}, b \in \mathbb{R}_{+}$ \\
\hline$\left(\begin{array}{ll}d & 0 \\
0 & 1\end{array}\right) d \in \mathbb{C}$ & $\left(\begin{array}{ll}1 & b \\
c & 0\end{array}\right) c \in \mathbb{C}, b \in \mathbb{R}_{+}$ \\
\hline$\left(\begin{array}{ll}1 & 0 \\
0 & d\end{array}\right) d \in \mathbb{C}$ & $\left(\begin{array}{ll}0 & b \\
c & 0\end{array}\right) c \in \mathbb{C}, b \in \mathbb{R}_{+}$ \\
\hline
\end{tabular}

In the above, $\mathbb{R}_{+}$represents the set of non-negative real numbers.

4.1. Simultaneously Diagonalizable Case. For the study of contractivity and complete contractivity in this situation we consider two possibilities. The first when $A_{1}$ and $A_{2}$ are simultaneously diagonalizable and the second when they are not. The simultaneously diagonalizable case reduces to the case of the bi-disc where we know that any contractive homomorphism is completely contractive. In all the other cases (when $A_{1}$ and $A_{2}$ are not simultaneously diagonalizable) we show that there exists a contractive homomorphism which is not completely contractive.

Consider first the case when $A_{1}$ and $A_{2}$ are simultaneously diagonalizable. Based on the discussion of linear equivalence above we need to study only the following possibilities:

TABLE 2. Simultaneously diagonalizable cases

\begin{tabular}{|c|c|}
\hline$A_{1}$ & $A_{2}$ \\
\hline \hline$\left(\begin{array}{ll}1 & 0 \\
0 & d\end{array}\right) d \in \mathbb{C}$ & $\left(\begin{array}{ll}1 & 0 \\
0 & 0\end{array}\right)$ \\
\hline$\left(\begin{array}{ll}d & 0 \\
0 & 1\end{array}\right) d \in \mathbb{C}$ & $\left(\begin{array}{ll}1 & 0 \\
0 & 0\end{array}\right)$ \\
\hline
\end{tabular}

Applying linear transformations as before, both cases can be reduced to $\mathbf{A}=\left(\left(\begin{array}{ll}1 & 0 \\ 0 & 0\end{array}\right),\left(\begin{array}{ll}0 & 0 \\ 0 & 1\end{array}\right)\right)$ which represents the bi-disc. As mentioned earlier, it is known that any contractive homomorphism is completely contractive in this case. We now study the situation when $A_{1}$ and $A_{2}$ are not simultaneously diagonalizable. 


\section{Contractivity, Complete Contractivity and Operator Space Structures}

We recall some notions about operator spaces which are relevant to our purpose.

Definition 5.1. (cf. [17, Chapter 13, 14]) An abstract operator space is a linear space $X$ together with a family of norms $\|\cdot\|_{k}$ defined on $\mathcal{M}_{k}(X), k=1,2,3, \ldots$, where $\|\cdot\|_{1}$ is simply a norm on the linear space $X$. These norms are required to satisfy the following compatibility conditions:

(1) $\|T \oplus S\|_{p+q}=\max \left\{\|T\|_{p},\|S\|_{q}\right\}$ and

(2) $\|A S B\|_{p} \leq\|A\|_{\mathrm{op}}\|S\|_{q}\|B\|_{\mathrm{op}}$

for all $S \in \mathcal{M}_{q}(X), T \in \mathcal{M}_{p}(X)$ and $A \in \mathcal{M}_{p, q}(\mathbb{C}), B \in \mathcal{M}_{q, p}(\mathbb{C})$.

Two such operator spaces $\left(X,\|\cdot\|_{k}\right)$ and $\left(Y,\|\cdot\|_{k}\right)$ are said to be completely isometric if there is a linear bijection $T: X \rightarrow Y$ such that $T \otimes I_{k}:\left(\mathcal{M}_{k}(X),\|\cdot\|_{k}\right) \rightarrow\left(\mathcal{M}_{k}(Y),\|\cdot\|_{k}\right)$ is an isometry for every $k \in \mathbb{N}$. Here we have identified $\mathcal{M}_{k}(X)$ with $X \otimes \mathcal{M}_{k}$ in the usual manner. We note that a normed linear space $(X,\|\cdot\|)$ admits an operator space structure if and only if there is an isometric embedding of it into the algebra of operators $\mathcal{B}(\mathcal{H})$ on some Hilbert space $\mathcal{H}$. This is the well-known theorem of Ruan (cf. [16]).

We recall here the notions of MIN and MAX operator spaces and a measure of their distance, $\alpha(X)$, following [17, Chapter 14].

Definition 5.2. The MIN operator structure $M I N(X)$ on a (finite dimensional) normed linear space $X$ is obtained by isometrically embedding $X$ in the $C^{*}$ algebra $C\left(\left(X^{*}\right)_{1}\right)$, of continuous functions on the unit ball $\left(X^{*}\right)_{1}$ of the dual space. Thus for $\left(\left(v_{i j}\right)\right)$ in $\mathcal{M}_{k}(X)$, we set

$$
\left\|\left(\left(v_{i j}\right)\right)\right\|_{M I N}=\left\|\left(\left(\widehat{v_{i j}}\right)\right)\right\|=\sup \left\{\left\|\left(\left(f\left(v_{i j}\right)\right)\right)\right\|: f \in\left(X^{*}\right)_{1}\right\},
$$

where the norm of a scalar matrix $\left(\left(f\left(v_{i j}\right)\right)\right)$ in $\mathcal{M}_{k}$ is the operator norm.

For an arbitrary $k \times k$ matrix over $X$, we simply write $\left\|\left(\left(v_{i j}\right)\right)\right\|_{M I N(X)}$ to denote its norm in $\mathcal{M}_{k}(X)$. This is the minimal way in which we represent the normed space as an operator space. There is also a 'maximal' representation which is denoted $M A X(X)$.

Definition 5.3. The operator space $M A X(X)$ is defined by setting

$$
\left\|\left(\left(v_{i j}\right)\right)\right\|_{M A X}=\sup \left\{\left\|\left(\left(T\left(v_{i j}\right)\right)\right)\right\|: T: X \rightarrow B(\mathcal{H})\right\},
$$

and the supremum is taken over all isometries $T$ and all Hilbert spaces $\mathcal{H}$.

Every operator space structure on a normed linear space $X$ 'lies between' $M I N(X)$ and $M A X(X)$. The extent to which the two operator space structures $M I N(X)$ and $M A X(X)$ differ is characterized by the constant $\alpha(X)$ introduced by Paulsen (cf.[17, Chapter 14]), which we recall below.

Definition 5.4. The constant $\alpha(X)$ is defined as

$$
\alpha(X)=\sup \left\{\left\|\left(\left(v_{i j}\right)\right)\right\|_{M A X}:\left\|\left(\left(v_{i j}\right)\right)\right\|_{M I N} \leq 1,\left(\left(v_{i j}\right)\right) \in \mathcal{M}_{k}(X), k \in \mathbb{N}\right\} .
$$

Thus $\alpha(X)=1$ if and only if the identity map is a complete isometry from $\operatorname{MIN}(X)$ to $M A X(X)$. Equivalently, we conclude that there exists a unique operator space structure on $X$ whenever $\alpha(X)$ is 1 . Therefore, those normed linear spaces for which $\alpha(X)=1$ are rather special. Unfortunately, there aren't too many of them! The familiar examples are $\left(\mathbb{C}^{2},\|\cdot\|_{\infty}\right)$, and consequently $\mathbb{C}^{2}$ with the $\ell_{1}$ norm. It is pointed out in [16, pp. 76]) that $\alpha(X)>1$ for $\operatorname{dim}(X) \geq 3$, refining an earlier result of Paulsen that $\alpha(X)>1$ whenever $\operatorname{dim}(X) \geq 5$. This leaves the question open for normed linear spaces whose dimension is 2 .

Returning to the space $\left(\mathbb{C}^{2},\|\cdot\|_{\mathbf{A}}\right)$ with $\left\|\left(z_{1}, z_{2}\right)\right\|_{\mathbf{A}}=\left\|z_{1} A_{1}+z_{2} A_{2}\right\|_{\text {op }}$, we show below that $\alpha\left(\Omega_{\mathbf{A}}\right)>1$ in a large number of cases. From [18, Theorem 4.2], it therefore follows that, in all these 
cases, there must exist a contractive homomorphism of $\mathcal{O}\left(\Omega_{\mathbf{A}}\right)$ into the algebra $B(\mathcal{H})$ which is not completely contractive. In the remaining cases, the existence of a contractive homomorphism which is not completely contractive is established by a careful study of certain extremal problems.

The norm $\left\|\left(z_{1}, z_{2}\right)\right\|_{\mathbf{A}}=\left\|z_{1} A_{1}+z_{2} A_{2}\right\|_{\text {op }}$ defines a natural isometric embedding into $\mathcal{M}_{2}(\mathbb{C})$ given by $\left(z_{1}, z_{2}\right) \mapsto z_{1} A_{1}+z_{2} A_{2}$. However, note that

$$
\left\|\left(z_{1}, z_{2}\right)\right\|_{\mathbf{A}}=\left\|z_{1} A_{1}+z_{2} A_{2}\right\|_{\mathrm{op}}=\left\|z_{1} A_{1}^{\mathrm{t}}+z_{2} A_{2}^{\mathrm{t}}\right\|_{\mathrm{op}}=\left\|\left(z_{1}, z_{2}\right)\right\|_{\mathbf{A}^{\mathrm{t}}} .
$$

We, therefore, get another isometric embedding into $\mathcal{M}_{2}(\mathbb{C})$ given by $\left(z_{1}, z_{2}\right) \mapsto z_{1} A_{1}^{\mathrm{t}}+z_{2} A_{2}^{\mathrm{t}}$.

In a variety of cases the operator spaces determined by these two embeddings are distinct and the parameter $\alpha>1$ in these cases. Therefore, the existence of contractive homomorphisms which are not completely contractive is established in these cases. We present the details below.

Recall the map $P_{\mathbf{A}}$ defined earlier by $P_{\mathbf{A}}\left(z_{1}, z_{2}\right)=z_{1} A_{1}+z_{2} A_{2}$. Let $P_{\mathbf{A}}^{(2)}=P_{\mathbf{A}} \otimes I_{2}$. For the three families of matrices $\mathbf{A}=\left(A_{1}, A_{2}\right)$ characterized in Table 1 we show that $\mathbf{A}$ and $\mathbf{A}^{\mathrm{t}}$ define distinct operator space structures unless $|d|=1$ or $b=|c|$.

Theorem 5.5. Let $Z_{1}=\left(\begin{array}{ll}1 & 0 \\ 0 & 0\end{array}\right)$ and $Z_{2}=\left(\begin{array}{ll}0 & 1 \\ 0 & 0\end{array}\right)$. If $|d| \neq 1$ and $b \neq|c|$ then $\left\|P_{\mathbf{A}}^{(2)}\left(Z_{1}, Z_{2}\right)\right\|_{\text {op }} \neq$ $\left\|P_{\mathbf{A}^{\mathrm{t}}}^{(2)}\left(Z_{1}, Z_{2}\right)\right\|_{\text {op }}$.

Proof. We illustrate the proof for the case $A_{1}=\left(\begin{array}{ll}1 & 0 \\ 0 & d\end{array}\right), A_{2}=\left(\begin{array}{ll}1 & b \\ c & 0\end{array}\right)$. The other cases can be proved similarly.

For this case

$$
\begin{aligned}
\left\|P_{\mathbf{A}}^{(2)}\left(Z_{1}, Z_{2}\right)\right\|_{\mathrm{op}}^{2} & =\left\|\left(\begin{array}{cc}
\left(Z_{1}+Z_{2}\right) & b Z_{2} \\
c Z_{2} & d Z_{1}
\end{array}\right)\left(\begin{array}{cc}
\left(Z_{1}+Z_{2}\right)^{*} & \bar{c} Z_{2}^{*} \\
b Z_{2}^{*} & \bar{d} Z_{1}^{*}
\end{array}\right)\right\|_{\mathrm{op}} \\
& =\left\|\left(\begin{array}{cc}
\left(Z_{1}+Z_{2}\right)\left(Z_{1}+Z_{2}\right)^{*}+b^{2} Z_{2} Z_{2}^{*} & \bar{c}\left(Z_{1}+Z_{2}\right) Z_{2}^{*}+b \bar{d} Z_{2} Z_{1}^{*} \\
c Z_{2}\left(Z_{1}+Z_{2}\right)^{*}+b d Z_{1} Z_{2}^{*} & |c|^{2} Z_{2} Z_{2}^{*}+|d|^{2} Z_{1} Z_{1}^{*}
\end{array}\right)\right\|_{\mathrm{op}} .
\end{aligned}
$$

Similarly we have

$$
\left\|P_{\mathbf{A}^{\mathrm{t}}}^{(2)}\left(Z_{1}, Z_{2}\right)\right\|_{\mathrm{op}}^{2}=\left\|\left(\begin{array}{cc}
\left(Z_{1}+Z_{2}\right)\left(Z_{1}+Z_{2}\right)^{*}+|c|^{2} Z_{2} Z_{2}^{*} & b\left(Z_{1}+Z_{2}\right) Z_{2}^{*}+c \bar{d} Z_{2} Z_{1}^{*} \\
b Z_{2}\left(Z_{1}+Z_{2}\right)^{*}+\bar{c} d Z_{1} Z_{2}^{*} & b^{2} Z_{2} Z_{2}^{*}+|d|^{2} Z_{1} Z_{1}^{*}
\end{array}\right)\right\|_{\mathrm{op}} .
$$

Assume $\left\|P_{\mathbf{A}}^{(2)}\left(Z_{1}, Z_{2}\right)\right\|_{\text {op }}^{2}=\left\|P_{\mathbf{A}^{\mathrm{t}}}^{(2)}\left(Z_{1}, Z_{2}\right)\right\|_{\text {op }}^{2}$. Using the form of $\left(Z_{1}, Z_{2}\right)$ this is equivalent to

$$
\left\|\left(\begin{array}{cc}
2+b^{2} & \bar{c} \\
c & |c|^{2}+|d|^{2}
\end{array}\right)\right\|_{\mathrm{op}}=\left\|\left(\begin{array}{cc}
2+|c|^{2} & b \\
b & b^{2}+|d|^{2}
\end{array}\right)\right\|_{\mathrm{op}}
$$

i.e. $\left(b^{2}-|c|^{2}\right)\left(1-|d|^{2}\right)=0$ (note that the matrices on the left and right have the same trace), from which the result follows.

Since $\alpha\left(\Omega_{\mathbf{A}}\right)=1$ if and only if the two operator spaces $\operatorname{MIN}\left(\Omega_{\mathbf{A}}\right)$ and $\operatorname{MAX}\left(\Omega_{\mathbf{A}}\right)$ are completely isometric, it follows from the Theorem we have just proved that if $|d| \neq 1$ and $b \neq|c|$, then $\alpha(X)>1$. Consequently, there exists a contractive homomorphism of $\mathcal{O}\left(\Omega_{\mathbf{A}}\right)$ into $B(\mathcal{H})$, which is not completely contractive.

Example 5.6. (Euclidean Ball) The Euclidean ball $\mathbb{B}^{2}$ is characterized by $A_{1}=\left(\begin{array}{ll}1 & 0 \\ 0 & 0\end{array}\right), A_{2}=\left(\begin{array}{ll}0 & 1 \\ 0 & 0\end{array}\right)$. So, in Theorem 5.5, we have $|d| \neq 1$ and $b \neq|c|$. Hence $\mathbf{A}$ and $\mathbf{A}^{\mathrm{t}}$ give rise to distinct operator space structures and, consequently, there exists a contractive homomorphism which is not completely contractive.

\section{Cases not Amenable to the Operator Space Method}

Theorem 5.5 shows that there is a contractive homomorphism which is not completely contractive for all the choices of $\left(A_{1}, A_{2}\right)$ listed in Table 1 except when $|d|=1$ or $b=|c|$. We are, therefore, left with the following families of $\left(A_{1}, A_{2}\right)$ to be considered: 
TABLE 3. Cases not covered by the operator space approach

\begin{tabular}{|c|c|c|}
\hline & $A_{1}$ & $A_{2}$ \\
\hline \hline (i) & $\left(\begin{array}{cc}1 & 0 \\
0 & e^{i \theta}\end{array}\right) \theta \in \mathbb{R}$ & $\left(\begin{array}{ll}1 & b \\
c & 0\end{array}\right) c \in \mathbb{C}, b \in \mathbb{R}_{+}$ \\
\hline (ii) & $\left(\begin{array}{cc}1 & 0 \\
0 & e^{i \theta}\end{array}\right) \theta \in \mathbb{R}$ & $\left(\begin{array}{cc}0 & b \\
c & 0\end{array}\right) c \in \mathbb{C}, b \in \mathbb{R}_{+}$ \\
\hline (iii) & $\left(\begin{array}{cc}e^{i \theta} & 0 \\
0 & 1\end{array}\right) \theta \in \mathbb{R}$ & $\left(\begin{array}{cc}1 & b \\
c & 0\end{array}\right) c \in \mathbb{C}, b \in \mathbb{R}_{+}$ \\
\hline (iv) & $\left(\begin{array}{cc}1 & 0 \\
0 & d\end{array}\right) d \in \mathbb{C}$ & $\left(\begin{array}{cc}1 & |c| \\
c & 0\end{array}\right) c \in \mathbb{C}$ \\
\hline (v) & $\left(\begin{array}{ll}1 & 0 \\
0 & d\end{array}\right) d \in \mathbb{C}$ & $\left(\begin{array}{cc}0 & |c| \\
c & 0\end{array}\right) c \in \mathbb{C}$ \\
\hline (vi) & $\left(\begin{array}{ll}d & 0 \\
0 & 1\end{array}\right) d \in \mathbb{C}$ & $\left(\begin{array}{cc}1 & |c| \\
c & 0\end{array}\right) c \in \mathbb{C}$ \\
\hline
\end{tabular}

These six families are not disjoint and have been classified as such on the basis of the method of proof used.

6.1. Dual norm method. We first consider a special case of type (ii) in Table 3 with $A_{1}=$ $\left(\begin{array}{ll}1 & 0 \\ 0 & 1\end{array}\right), A_{2}=\left(\begin{array}{ll}0 & 1 \\ 0 & 0\end{array}\right)$. Although this case is covered by the more general method to be outlined later we present an alternate, interesting procedure for this example since it is possible to explicitly calculate the dual norm $\|\cdot\|_{\mathbf{A}}^{*}$ in this case. Equipped with the information about the dual norm we can directly construct a pair $\mathbf{V}=\left(V_{1}, V_{2}\right)$ such that $\left\|L_{\mathbf{V}}\right\| \leq 1$ and $\left\|L_{\mathbf{V}}^{(2)}\left(P_{\mathbf{A}}\right)\right\|>1$.

Note that in this case

$$
\left\|\left(z_{1}, z_{2}\right)\right\|_{\mathbf{A}}=\frac{\left|z_{2}\right|+\sqrt{\left|z_{2}\right|^{2}+4\left|z_{1}\right|^{2}}}{2}
$$

and the unit ball

$$
\Omega_{\mathbf{A}}=\left\{\left(z_{1}, z_{2}\right):\left|z_{1}\right|^{2}+\left|z_{2}\right|<1\right\} .
$$

Lemma 6.1. Let $A_{1}=\left(\begin{array}{ll}1 & 0 \\ 0 & 1\end{array}\right), A_{2}=\left(\begin{array}{ll}0 & 1 \\ 0 & 0\end{array}\right)$. If $\left(\omega_{1}, \omega_{2}\right) \in\left(\mathbb{C}^{2},\|\cdot\|_{\mathbf{A}}^{*}\right)$ then the dual norm

$$
\left\|\left(\omega_{1}, \omega_{2}\right)\right\|_{\mathbf{A}}^{*}= \begin{cases}\frac{\left|\omega_{1}\right|^{2}+4\left|\omega_{2}\right|^{2}}{4\left|\omega_{2}\right|} & \text { if }\left|\omega_{2}\right| \geq \frac{\left|\omega_{1}\right|}{2} ; \\ \left|\omega_{1}\right| & \text { if }\left|\omega_{2}\right| \leq \frac{\left|\omega_{1}\right|}{2} .\end{cases}
$$

Proof. Let $f_{\omega_{1}, \omega_{2}}$ be the linear functional on $\left(\mathbb{C}^{2},\|\cdot\|_{\mathbf{A}}\right)$ defined by

$$
f_{\omega_{1}, \omega_{2}}\left(z_{1}, z_{2}\right)=\omega_{1} z_{1}+\omega_{2} z_{2}
$$


Then

$$
\begin{aligned}
\left\|\left(\omega_{1}, \omega_{2}\right)\right\|_{\mathbf{A}}^{*} & =\sup _{\left(z_{1}, z_{2}\right) \in \Omega_{\mathbf{A}}}\left|f_{\omega_{1}, \omega_{2}}\left(z_{1}, z_{2}\right)\right| \\
& =\sup _{\left|z_{2}\right| \leq 1-\left|z_{1}\right|^{2}}\left|\omega_{1} z_{1}+\omega_{2} z_{2}\right| \\
& =\sup _{\left|z_{2}\right| \leq 1-\left|z_{1}\right|^{2}}\left(\left|\omega_{1}\right|\left|z_{1}\right|+\left|\omega_{2}\right|\left|z_{2}\right|\right) \\
& =\sup _{\left|z_{1}\right| \leq 1}\left(\left|\omega_{1}\right|\left|z_{1}\right|+\left|\omega_{2}\right|\left(1-\left|z_{1}\right|^{2}\right)\right) .
\end{aligned}
$$

If $\left|\omega_{2}\right| \geq \frac{\left|\omega_{1}\right|}{2}$ the expression on the right attains its maximum at $\left|z_{1}\right|=\frac{\left|\omega_{1}\right|}{2\left|\omega_{2}\right|} \leq 1$ and the maximum value is $\frac{\left|\omega_{1}\right|^{2}+4\left|\omega_{2}\right|^{2}}{4\left|\omega_{2}\right|}$.

If $\left|\omega_{2}\right| \leq \frac{\left|\omega_{1}\right|}{2}$ the expression on the right is monotonic in $\left|z_{1}\right|$ and the maximum is attained at $\left|z_{1}\right|=1$. The maximum value in this case is $\left|\omega_{1}\right|$.

Theorem 6.2. Let $A_{1}=\left(\begin{array}{ll}1 & 0 \\ 0 & 1\end{array}\right), A_{2}=\left(\begin{array}{ll}0 & 1 \\ 0 & 0\end{array}\right)$ and $V_{1}=\left(\begin{array}{ll}\frac{1}{\sqrt{2}} & 0\end{array}\right), V_{2}=\left(\begin{array}{ll}0 & 1\end{array}\right)$. Then

$$
\begin{aligned}
& \text { (i) }\left\|L_{\mathbf{V}}\right\|_{\left(\mathbb{C}^{2},\|\cdot\|_{\mathbf{A}}^{*}\right) \rightarrow\left(\mathbb{C}^{2},\|\cdot\|_{2}\right)}=1 \\
& \text { (ii) }\left\|L_{\mathbf{V}}^{(2)}\left(P_{\mathbf{A}}\right)\right\|=\sqrt{\frac{3}{2}} .
\end{aligned}
$$

Consequently $\rho_{\mathbf{V}}$, for this choice of $\mathbf{V}=\left(V_{1}, V_{2}\right)$, is contractive on $\mathcal{O}\left(\Omega_{\mathbf{A}}\right)$ but not completely contractive.

Proof of (i).

$$
\begin{aligned}
\left\|L_{\mathbf{V}}\right\|_{\left(\mathbb{C}^{2},\|\cdot\|_{\mathbf{A}}^{*}\right) \rightarrow\left(\mathbb{C}^{2},\|\cdot\|_{2}\right)}^{2} & =\sup _{\left\|\left(\omega_{1}, \omega_{2}\right)\right\|_{\mathbf{A}}^{*}=1}\left\|\omega_{1} V_{1}+\omega_{2} V_{2}\right\|_{2}^{2} \\
& =\sup _{\left\|\left(\omega_{1}, \omega_{2}\right)\right\|_{\mathbf{A}}^{*}=1}\left(\frac{\left|\omega_{1}\right|^{2}}{2}+\left|\omega_{2}\right|^{2}\right) .
\end{aligned}
$$

We now consider two cases:

Case (a): $\left|\omega_{2}\right| \geq \frac{\left|\omega_{1}\right|}{2}$ and $1=\left\|\left(\omega_{1}, \omega_{2}\right)\right\|_{\mathbf{A}}^{*}=\frac{\left|\omega_{1}\right|^{2}+4\left|\omega_{2}\right|^{2}}{4\left|\omega_{2}\right|}$ from Lemma 6.1]

These two constraints together can be seen to be equivalent to the constraints $\frac{1}{2} \leq\left|\omega_{2}\right| \leq 1$ and $\left|\omega_{1}\right|^{2}=4\left|\omega_{2}\right|\left(1-\left|\omega_{2}\right|\right)$.

Hence the supremum above for this range of $\left(\omega_{1}, \omega_{2}\right)$ is given by

$$
\sup _{\frac{1}{2} \leq\left|\omega_{2}\right| \leq 1}\left|\omega_{2}\right|\left(2-\left|\omega_{2}\right|\right)=1
$$

Case (b): $\left|\omega_{2}\right| \leq \frac{\left|\omega_{1}\right|}{2}$ and $1=\left\|\left(\omega_{1}, \omega_{2}\right)\right\|_{\mathbf{A}}^{*}=\left|\omega_{1}\right|$ from Lemma 6.1.

The supremum for this range of $\left(\omega_{1}, \omega_{2}\right)$ is given by

$$
\sup _{\left|\omega_{2}\right| \leq \frac{1}{2}}\left(\frac{1}{2}+\left|\omega_{2}\right|^{2}\right)=\frac{3}{4} .
$$

Taking the larger of the supremums in Case (a) and Case (b) we get that $\left\|L_{\mathbf{V}}\right\|=1$. 
Proof of (ii).

$$
\begin{aligned}
\left\|L_{\mathbf{V}}^{(2)}\left(P_{\mathbf{A}}\right)\right\|^{2} & =\left\|A_{1} \otimes V_{1}+A_{2} \otimes V_{2}\right\|^{2} \\
& =\left\|\left(\begin{array}{ll}
\frac{1}{\sqrt{2}} A_{1} & A_{2}
\end{array}\right)\right\|^{2} \\
& =\left\|\left(\begin{array}{ll}
\frac{1}{\sqrt{2}} A_{1} & A_{2}
\end{array}\right)\left(\begin{array}{c}
\frac{1}{\sqrt{2}} A_{1}^{*} \\
A_{2}^{*}
\end{array}\right)\right\| \\
& =\left\|\left(\begin{array}{ll}
\frac{3}{2} & 0 \\
0 & \frac{1}{2}
\end{array}\right)\right\| \text { using the form of } A_{1}, A_{2} \\
& =\frac{3}{2} .
\end{aligned}
$$

6.2. General cases not amenable to the operator space method. The various families of $\left(A_{1}, A_{2}\right)$ listed in Table 3 require a case by case analysis to show that there is a contractive homomorpism which is not completely contractive. We first present a general outline of the method used.

We choose the pair $\mathbf{V}=\left(V_{1}, V_{2}\right)$ to be of the form $V_{1}=\left(\begin{array}{ll}u & 0\end{array}\right), V_{2}=\left(\begin{array}{ll}0 & v\end{array}\right), u, v \in \mathbb{R}_{+}$. $L_{\mathbf{V}}:\left(\mathbb{C}^{2},\|\cdot\|_{\mathbf{A}}^{*}\right) \rightarrow\left(\mathbb{C}^{2},\|\cdot\|_{2}\right)$ then becomes the linear map $\left(z_{1}, z_{2}\right) \mapsto\left(z_{1} u, z_{2} v\right)$.

We show, in each case, that by a suitable choice of $u$ and $v$ we can ensure that $L_{\mathbf{V}}$ is contractive while $\left\|L_{\mathbf{V}}^{(2)}\left(P_{\mathbf{A}}\right)\right\|>1$ although $\left\|P_{\mathbf{A}}\right\|=1$ by definition.

We list the contractivity conditions (see Propositions 2.3 and 2.4 for details).

(a) $L_{\mathbf{V}}$ is contractive if and only if the following two conditions are satisfied:

(i) $u \leq \frac{1}{\left\|A_{1}^{*}\right\|}$ or $v \leq \frac{1}{\left\|A_{2}^{*}\right\|}$ and

$$
\inf _{\beta \in \mathbb{C}^{2},\|\beta\|=1}\left\{1-u^{2}\left\|A_{1}^{*} \beta\right\|^{2}-v^{2}\left\|A_{2}^{*} \beta\right\|^{2}+u^{2} v^{2}\left(\left\|A_{1}^{*} \beta\right\|^{2}\left\|A_{2}^{*} \beta\right\|^{2}-\left|\left\langle A_{1} A_{2}^{*} \beta, \beta\right\rangle\right|^{2}\right)\right\} \geq 0 .
$$

(b) $\left\|L_{\mathbf{V}}^{(2)}\left(P_{\mathbf{A}}\right)\right\| \leq 1$ if and only if

$$
\inf _{\beta \in \mathbb{C}^{2},\|\beta\|=1}\left\{1-u^{2}\left\|A_{1}^{*} \beta\right\|^{2}-v^{2}\left\|A_{2}^{*} \beta\right\|^{2}\right\} \geq 0
$$

Note that the term in parenthesis in (6.1) is non-negative by the Schwarz inequality and that the expression (6.2) is the same as the first three terms in (6.1).

We show that, in each case, we can choose $(u, v)$ such that the infimum in (6.1) is exactly 0 . Also that this infimum is attained at $\beta=\beta_{0}$ such that the term in parenthesis in (6.1) is positive (that is, the Schwarz inequality referred to above is a strict inequality at $\beta_{0}$ ). It then follows that the expression in braces in (6.2) is negative when $\beta=\beta_{0}$ and, consequently, the infimum in (6.2) is negative. Taken together it follows that $L_{\mathbf{V}}$ (and consequently $\rho_{\mathbf{V}}$ ) is contractive but $\left\|L_{\mathbf{V}}^{(2)}\left(P_{\mathbf{A}}\right)\right\|>1$ and, as a result, $\rho_{\mathbf{V}}^{(2)}$ is not contractive.

Let $\eta^{(i)}, i=1,2$, be the vectors such that $A_{1}^{*} \eta^{(i)}$ and $A_{2}^{*} \eta^{(i)}$ are linearly dependent. That is, the term in parenthesis in (6.1) vanishes when $\beta=\eta^{(i)}$.

We now provide the details of the argument which proceeds in two steps.

Step 1: Show that there are certain ranges of the parameters $(u, v)$ such that the infimum in (6.1) is not attained at $\eta^{(1)}$ or $\eta^{(2)}$ for those values of $(u, v)$. 
Let

$$
g_{u, v}(\beta)=1-u^{2}\left\|A_{1}^{*} \beta\right\|^{2}-v^{2}\left\|A_{2}^{*} \beta\right\|^{2}+u^{2} v^{2}\left(\left\|A_{1}^{*} \beta\right\|^{2}\left\|A_{2}^{*} \beta\right\|^{2}-\left|\left\langle A_{1} A_{2}^{*} \beta, \beta\right\rangle\right|^{2}\right) .
$$

We need to show that there exists $\beta$ such that

$$
g_{u, v}(\beta)<g_{u, v}\left(\eta^{(i)}\right), i=1,2,
$$

when $(u, v)$ take values in a range of interest. That is, we need to find $\beta$ such that

$$
g_{u, v}\left(\eta^{(i)}\right)-g_{u, v}(\beta)=a_{i}(\beta) u^{2}+b_{i}(\beta) v^{2}-c(\beta) u^{2} v^{2}>0
$$

Here

$$
\begin{aligned}
a_{i}(\beta) & =\left\|A_{1}^{*} \beta\right\|^{2}-\left\|A_{1}^{*} \eta^{(i)}\right\|^{2} \\
b_{i}(\beta) & =\left\|A_{2}^{*} \beta\right\|^{2}-\left\|A_{2}^{*} \eta^{(i)}\right\|^{2} \\
c(\beta) & =\left\|A_{1}^{*} \beta\right\|^{2}\left\|A_{2}^{*} \beta\right\|^{2}-\left|\left\langle A_{1} A_{2}^{*} \beta, \beta\right\rangle\right|^{2} \geq 0 .
\end{aligned}
$$

Consider the functions

$$
f_{i}(u, v, \beta)=a_{i}(\beta) u^{2}+b_{i}(\beta) v^{2}-c(\beta) u^{2} v^{2} \text { with } c(\beta) \geq 0, i=1,2 .
$$

The following result is evident from the nature of the functions $f_{i}(u, v, \beta)$.

Lemma 6.3. (i) Assume $a_{i}(\beta)>0$ for some fixed $\beta$ and $i=1,2$. Then, given any $u_{0}>0$, there exists $v_{0}>0$ (depending on $u_{0}$ ) such that $f_{i}(u, v, \beta)>0$ in the region $u<u_{0}, v<\frac{v_{0}}{u_{0}} u$, that is, inside the triangle with vertices $(0,0),\left(u_{0}, 0\right)$ and $\left(u_{0}, v_{0}\right)$.

(ii) Assume $b_{i}(\beta)>0$ for some fixed $\beta$ and $i=1,2$. Then, given any $v_{0}>0$, there exists $u_{0}>0$ (depending on $v_{0}$ ) such that $f_{i}(u, v, \beta)>0$ in the region $v<v_{0}, u<\frac{u_{0}}{v_{0}} v$, that is, inside the triangle with vertices $(0,0),\left(0, v_{0}\right)$ and $\left(u_{0}, v_{0}\right)$.

(iii) If $f_{i}\left(u_{0}, v_{0}, \beta\right)>0$ then $f_{i}\left(t u_{0}, t v_{0}, \beta\right)>0$ for $0<t<1$.

We will show below that, in each of the six cases in Table 3 , it is possible to ensure the positivity of $a_{i}(\beta), i=1,2$ or $b_{i}(\beta), i=1,2$ for some choice of $\beta$. Consequently, it will follow that the inequality (6.2) will be true for that vector $\beta$ with $(u, v)$ in the region characterized in Lemma 6.3 above. Hence, for $(u, v)$ in this range, the infimum is not attained at $\eta^{(i)}, i=1,2$.

Consider first the cases (i), (ii) and (iii).

\begin{tabular}{|c|c|c|}
\hline & $A_{1}$ & $A_{2}$ \\
\hline \hline (i) & $\left(\begin{array}{cc}1 & 0 \\
0 & e^{i \theta}\end{array}\right) \theta \in \mathbb{R}$ & $\left(\begin{array}{ll}1 & b \\
c & 0\end{array}\right) c \in \mathbb{C}, b \in \mathbb{R}_{+}$ \\
\hline (ii) & $\left(\begin{array}{cc}1 & 0 \\
0 & e^{i \theta}\end{array}\right) \theta \in \mathbb{R}$ & $\left(\begin{array}{ll}0 & b \\
c & 0\end{array}\right) c \in \mathbb{C}, b \in \mathbb{R}_{+}$ \\
\hline (iii) & $\left(\begin{array}{cc}e^{i \theta} & 0 \\
0 & 1\end{array}\right) \theta \in \mathbb{R}$ & $\left(\begin{array}{ll}1 & b \\
c & 0\end{array}\right) c \in \mathbb{C}, b \in \mathbb{R}_{+}$ \\
\hline
\end{tabular}

We use the unitary equivalence described in Section 4. In cases (i) and (ii) multiply $A_{1}$ and $A_{2}$ on the left by the unitary matrix $\left(\begin{array}{ll}1 & 0 \\ 0 & e^{-i \theta}\end{array}\right)$ so that $A_{1}$ becomes the identity matrix. In case (iii) 
multiply $A_{1}$ and $A_{2}$ on the left by the unitary matrix $\left(\begin{array}{cc}e^{-i \theta} & 0 \\ 0 & 1\end{array}\right)$ so that $A_{1}$ becomes the identity matrix.

Now conjugate $A_{1}$ and $A_{2}$ by the unitary which makes $A_{2}$ upper triangular so that cases (i),(ii) and (iii) reduce to the situation

$$
A_{1}=\left(\begin{array}{ll}
1 & 0 \\
0 & 1
\end{array}\right) \text { and } A_{2}=\left(\begin{array}{cc}
\mu & \sigma \\
0 & \nu
\end{array}\right) \text { with }|\mu| \geq|\nu|, \sigma \neq 0
$$

In this case $a_{i}(\beta)=0$ for all $\beta$ but it is possible to choose $\beta$ such that $b_{i}(\beta)>0$.

$\eta^{(i)}$ satisfies the equation $\left(A_{2}^{*}-\lambda_{i} A_{1}^{*}\right) \eta^{(i)}=0$. So in this case $\eta^{(i)}$ is a (unit) eigenvector of $A_{2}^{*}$ with eigenvalue $\lambda_{i}$. Since the eigenvalues of $A_{2}^{*}$ are $\bar{\mu}$ and $\bar{\nu}$ it follows that $\left\|A_{2}^{*} \eta^{(i)}\right\|^{2}=|\mu|^{2}$ or $|\nu|^{2}$. Hence we can take $\beta=\left(\begin{array}{l}1 \\ 0\end{array}\right)$ so that $b_{i}(\beta) \geq|\sigma|^{2}>0$.

Now consider cases (iv) and (v).

\begin{tabular}{|c|c|c|}
\hline & $A_{1}$ & $A_{2}$ \\
\hline \hline (iv) & $\left(\begin{array}{ll}1 & 0 \\
0 & d\end{array}\right) d \in \mathbb{C},|d| \neq 1$ & $\left(\begin{array}{cc}1 & |c| \\
c & 0\end{array}\right) c \in \mathbb{C}$ \\
\hline (v) & $\left(\begin{array}{ll}1 & 0 \\
0 & d\end{array}\right) d \in \mathbb{C},|d| \neq 1$ & $\left(\begin{array}{cc}0 & |c| \\
c & 0\end{array}\right) c \in \mathbb{C}$ \\
\hline
\end{tabular}

In cases (iv) and (v) we have, in Equation (6.4),

$$
\begin{aligned}
a_{i}(\beta) & =\left|\beta_{1}\right|^{2}+|d|^{2}\left|\beta_{2}\right|^{2}-\left|\eta_{1}^{(i)}\right|^{2}-|d|^{2}\left|\eta_{2}^{(i)}\right|^{2} \\
& =\left(\left|\beta_{1}\right|^{2}-\left|\eta_{1}^{(i)}\right|^{2}\right)+|d|^{2}\left(\left|\beta_{2}\right|^{2}-\left|\eta_{2}^{(i)}\right|^{2}\right) \\
& =\left(1-|d|^{2}\right)\left(\left|\eta_{2}^{(i)}\right|^{2}-\left|\beta_{2}\right|^{2}\right) .
\end{aligned}
$$

If $\left|\eta_{2}^{(i)}\right|=0$ or 1 then $c=0$ and it reduces to the simultaneously diagonalizable case. If $\left|\eta_{2}^{(i)}\right| \neq 0,1$ we can choose $\beta$ such that $\left|\beta_{2}\right|<\left|\eta_{2}^{(i)}\right|$ (resp. $\left|\beta_{2}\right|>\left|\eta_{2}^{(i)}\right|$ ) if $|d|<1$ (resp. $|d|>1$ ) to ensure that $a_{i}(\beta)>0$ for $i=1,2$.

The methods used in cases (iv) and (v) can be adapted to the last case (vi):

\begin{tabular}{|c|c|c|}
\hline & $A_{1}$ & $A_{2}$ \\
\hline \hline (vi) & $\left(\begin{array}{ll}d & 0 \\
0 & 1\end{array}\right) d \in \mathbb{C},|d| \neq 1$ & $\left(\begin{array}{cc}1 & |c| \\
c & 0\end{array}\right) c \in \mathbb{C}$ \\
\hline
\end{tabular}

Step 2: Show that, in each case, there is a choice of $(u, v)$ in the region characterized in Lemma 6.3 for which the infimum in (6.1) is, in fact, zero.

We choose $\hat{\beta}$ to ensure that $a_{i}(\hat{\beta})$ or $b_{i}(\hat{\beta})$ is positive as described in Step 1 .

Note that $g_{u, v}(\hat{\beta})$ vanishes at the two points $(u, v)=\left(\frac{1}{\left\|A_{1}^{*}(\hat{\beta})\right\|}, 0\right),(u, v)=\left(0, \frac{1}{\left\|A_{2}^{*}(\hat{\beta})\right\|}\right)$ and also along a curve joining these two points.

We now consider two cases:

Case $(\mathbf{i}): a_{i}(\hat{\beta})>0$

Choose $\left(u_{0}, v_{0}\right)$ such that $0<v_{0}<\frac{1}{\left\|A_{2}^{*}\right\|}, f_{i}\left(u_{0}, v_{0}, \hat{\beta}\right)>0$ and $g_{u_{0}, v_{0}}(\hat{\beta})=0$. This is possible using Lemma 6.3 and the above note about the vanishing of $g_{u, v}(\hat{\beta})$. 
Let

$$
x_{0}=\inf \left\{u: \inf _{\beta} g_{u, \lambda_{0} u}(\beta) \leq 0\right\} \text { where } \lambda_{0}=\frac{v_{0}}{u_{0}} .
$$

Note that $x_{0}^{2} \geq \frac{1}{\left\|A_{1}^{*}\right\|^{2}+\lambda_{0}^{2}\left\|A_{2}^{*}\right\|^{2}}$. Also, from Lemma 6.3, it is clear that $f_{i}\left(x_{0}, \lambda_{0} x_{0}, \hat{\beta}\right)>0$.

We now show that $\inf _{\beta} g_{x_{0}, \lambda_{0} x_{0}}(\beta)=0$.

To prove this we first show that $g_{\left(x_{0}, \lambda_{0} x_{0}\right)}(\beta) \geq 0$ for all $\beta$ (with $\|\beta\|_{2}=1$ ) as follows. Assume there exists $\beta=\mu$ such that $g_{\left(x_{0}, \lambda_{0} x_{0}\right)}(\mu)<0$. Then there exists a neighborhood $U$ of $x_{0}$ such that $g_{u, \lambda_{0} u}(\mu)<0$ for all $u \in U$. For any $u \in U, \inf _{\beta} g_{u, \lambda_{0} u}(\beta)<0$, since $g_{u, \lambda_{0} u}(\mu)<0$ for all $u \in U$. Since $U$ is a neighborhood of $x_{0}$ there exists a $u \in U$ such that $u<x_{0}$. By the previous assertion, $\inf _{\beta} g_{u, \lambda_{0} u}(\beta) \leq 0$ for this smaller value of $u$, which is a contradiction.

Since $\inf _{\beta} g_{x_{0}, \lambda_{0} x_{0}}(\beta) \leq 0$ by the definition of $x_{0}$ it follows that $\inf _{\beta} g_{x_{0}, \lambda_{0} x_{0}}(\beta)=0$.

Case (ii): $b_{i}(\hat{\beta})>0$

The arguments in this case are similar to Case (i). This time choose $\left(u_{0}, v_{0}\right)$ such that $0<$ $u_{0}<\frac{1}{\left\|A_{1}^{*}\right\|}, f_{i}\left(u_{0}, v_{0}, \hat{\beta}\right)>0$ and $g_{u_{0}, v_{0}}(\hat{\beta})=0$.

Let

$$
y_{0}=\inf \left\{v: \inf _{\beta} g_{\lambda_{0} v, v}(\beta) \leq 0\right\} \text { where } \lambda_{0}=\frac{u_{0}}{v_{0}} .
$$

As in Case (i) we can see that $y_{0}^{2} \geq \frac{1}{\lambda_{0}^{2}\left\|A_{1}^{*}\right\|^{2}+\mid A_{2}^{*} \|^{2}}$ and (from Lemma 6.3) that $f_{i}\left(\lambda_{0} y_{0}, y_{0}, \hat{\beta}\right)>0$.

Using a procedure similar to that used in Case (i) it follows that $\inf _{\beta} g_{\lambda_{0} y_{0}, y_{0}}(\beta)=0$.

We have therefore shown that for all the cases in Table 3 which were not covered by the operator space approach it is possible to choose $(u, v)$ such that the infimum in (6.1) is zero and this infimum is attained at a vector $\beta$ not equal to $\eta^{(1)}$ or $\eta^{(2)}$, so that the last term in parenthesis in (6.1) is positive at $\beta$.

It follows that, in each of these cases, there exists a contractive homomorphism which is not completely contractive.

\section{An Interesting Operator Space Computation}

In Section 5 the existence of contractive homomorphisms which are not completely contractive was shown in many cases by studying different isometric embeddings of the space $\left(\mathbb{C}^{2},\|\cdot\|_{\mathbf{A}}\right)$ into $\left(\mathcal{M}_{2},\|\cdot\|_{\text {op }}\right)$ which led to distinct operator space structures. The two embeddings considered there were $\left(z_{1}, z_{2}\right) \mapsto z_{1} A_{1}+z_{2} A_{2}$ and $\left(z_{1}, z_{2}\right) \mapsto z_{1} A_{1}^{\mathrm{t}}+z_{2} A_{2}^{\mathrm{t}}$. In this section we show that we can, for some choices of $\left(A_{1}, A_{2}\right)$, construct large collections of isometric embeddings of the space $\left(\mathbb{C}^{2},\|\cdot\|_{\mathbf{A}}\right)$ into various matrix spaces. Although the embeddings are into very distinct matrix spaces, we show that the operator space structures thus obtained are equivalent.

A result which is very useful in this context is the following proposition due to Douglas, Muhly and Pearcy (cf. [7, Prop. 2.2]).

Proposition 7.1. For $i=1,2$, let $T_{i}$ be a contraction on a Hilbert space $\mathcal{H}_{i}$ and let $X$ be an operator mapping $\mathcal{H}_{2}$ into $\mathcal{H}_{1}$. A necessary and sufficient condition that the operator on $\mathcal{H}_{1} \oplus \mathcal{H}_{2}$ defined by the matrix $\left(\begin{array}{cc}T_{1} & X \\ 0 & T_{2}\end{array}\right)$ be a contraction is that there exist a contraction $C$ mapping $\mathcal{H}_{2}$ into $\mathcal{H}_{1}$ such that

$$
X=\sqrt{1_{\mathcal{H}_{1}}-T_{1} T_{1}^{*}} C \sqrt{1_{\mathcal{H}_{2}}-T_{2}^{*} T_{2}} .
$$

The operator norm of the block matrix $\left(\begin{array}{cc}\alpha I_{m} & B \\ 0 & \alpha I_{n}\end{array}\right)$, where $B$ is an $m \times n$ matrix and $\alpha \in \mathbb{C}$, is not hard to compute (cf. [10, Lemma 2.1]). The result can be easily extended to a matrix of the form $\left(\begin{array}{cc}\alpha_{1} I_{m} & B \\ 0 & \alpha_{2} I_{n}\end{array}\right)$, for arbitrary $\alpha_{1}, \alpha_{2} \in \mathbb{C}$. 
Lemma 7.2. If $B$ is an $m \times n$ matrix and $\alpha_{1}, \alpha_{2} \in \mathbb{C}$ then

$$
\left\|\left(\begin{array}{cc}
\alpha_{1} I_{m} & B \\
0 & \alpha_{2} I_{n}
\end{array}\right)\right\|=\left\|\left(\begin{array}{cc}
\alpha_{1} & \|B\| \\
0 & \alpha_{2}
\end{array}\right)\right\|
$$

Proof. Consider the following two sets

$$
S_{1}=\left\{\left(\left(\alpha_{1}, \alpha_{2}\right) ; B\right):\left\|\left(\begin{array}{cc}
\alpha_{1} I_{m} & B \\
0 & \alpha_{2} I_{n}
\end{array}\right)\right\| \leq 1\right\}
$$

and

$$
S_{2}=\left\{\left(\left(\alpha_{1}, \alpha_{2}\right) ; B\right):\left\|\left(\begin{array}{cc}
\alpha_{1} & \|B\| \\
0 & \alpha_{2}
\end{array}\right)\right\| \leq 1\right\} .
$$

To prove the lemma, it is sufficient to show that these unit balls are the same.

From Proposition 7.1 the condition for the contractivity of the elements of $S_{1}$ and $S_{2}$ is the same, that is,

$$
\|B\|^{2} \leq\left(1-\left|\alpha_{1}\right|^{2}\right)\left(1-\left|\alpha_{2}\right|^{2}\right)
$$

The important observation from the lemma above is that, for fixed $\alpha_{1}, \alpha_{2}$, the norm of the matrix $\left(\begin{array}{cc}\alpha_{1} I_{m} & B \\ 0 & \alpha_{2} I_{n}\end{array}\right)$ depends only on $\|B\|$.

Now consider the pair $\mathbf{A}=\left(A_{1}, A_{2}\right)$ with $A_{1}=\left(\begin{array}{cc}\alpha_{1} & 0 \\ 0 & \alpha_{2}\end{array}\right), A_{2}=\left(\begin{array}{cc}0 & \beta \\ 0 & 0\end{array}\right)$. Given any $m \times n$ matrix $B$ with $\|B\|=|\beta|$ we have the following isometric embedding of $\left(\mathbb{C}^{2},\|\cdot\|_{\mathbf{A}}\right)$ into $\left(\mathcal{M}_{m+n},\|\cdot\|_{\text {op }}\right)$

$$
\left(z_{1}, z_{2}\right) \mapsto\left(\begin{array}{cc}
z_{1} \alpha_{1} I_{m} & z_{2} B \\
0 & z_{1} \alpha_{2} I_{n}
\end{array}\right)
$$

For various choices of the dimensions $m, n$ and the matrix $B$, this represents a large collection of isometric embeddings.

For fixed $\alpha_{1}, \alpha_{2}$, we let $X_{B}$ represent the above embedding of $\left(\mathbb{C}^{2},\|\cdot\|_{\mathbf{A}}\right)$ into $\left(\mathcal{M}_{m+n},\|\cdot\|_{\text {op }}\right)$. We now show that the operator space structures determined by these embeddings depend only on $\|B\|$. If $\mathcal{V}_{\mathbf{A}}$ is the space $\left(\mathbb{C}^{2},\|\cdot\|_{\mathbf{A}}\right)$, then $\left(X_{B} \otimes I_{k}\right)$ gives the embedding of $\mathcal{M}_{k}\left(\mathcal{V}_{\mathbf{A}}\right)$ into $\mathcal{M}_{k}\left(\mathcal{M}_{m+n}(\mathbb{C})\right)$. An element of $\mathcal{M}_{k}\left(\mathcal{V}_{\mathbf{A}}\right)$ is defined by a pair of $k \times k$ matrices $Z_{1}, Z_{2}$, and the corresponding embedding into $\mathcal{M}_{k}\left(\mathcal{M}_{m+n}(\mathbb{C})\right)$ has the form

$$
\left(\begin{array}{cc}
\alpha_{1} Z_{1} \otimes I_{m} & Z_{2} \otimes B \\
0 & \alpha_{2} Z_{1} \otimes I_{n}
\end{array}\right) .
$$

It now remains to show that the operator norm of this matrix depends only on $\|B\|$. Using Proposition 7.1 it can be shown that

$$
\left\|\left(\begin{array}{cc}
\alpha_{1} Z_{1} \otimes I_{m} & Z_{2} \otimes B \\
0 & \alpha_{2} Z_{1} \otimes I_{n}
\end{array}\right)\right\| \leq 1 \text { if and only if }\left\|\left(\begin{array}{cc}
\alpha_{1} Z_{1} & Z_{2}\|B\| \\
0 & \alpha_{2} Z_{1}
\end{array}\right)\right\| \leq 1 .
$$

Hence it follows that these two norms are in fact equal. We have therefore proved the following theorem.

Theorem 7.3. For all $m \times n$ matrices $B$ with the same (operator) norm, the operator space structures on $\mathbb{C}^{m+n}$, determined by the different embeddings

$$
\left(z_{1}, z_{2}\right) \mapsto z_{1}\left(\begin{array}{cc}
\alpha_{1} I_{m} & 0 \\
0 & \alpha_{2} I_{n}
\end{array}\right)+z_{2}\left(\begin{array}{cc}
0 & B \\
0 & 0
\end{array}\right), \alpha_{1}, \alpha_{2} \in \mathbb{C},
$$

are completely isometric irrespective of the particular choice of B. Moreover all of them are completely isometric to the MIN space.

Acknowledgment. The authors gratefully acknowledge the help they have received from Sayan Bagchi, Michael Dritschel and Dmitry Yakubovich. 


\section{REFERENCES}

[1] W. Arveson, Subalgebras of $C^{*}$-algebras II, Acta Math., 128 (1972), 271 - 308.

[2] J. Agler, Rational dilation on an annulus, Ann. of Math., 121 (1985), 537 - 563.

[3] J. Agler and N. J. Young, Operators having the symmetrized bidisc as a spectral set, Proc. Edinburgh Math. Soc., 43 (2000), $195-210$.

[4] B. Bagchi and G. Misra, Contractive homomorphisms and tensor product norms, Integral Equations and Operator Theory, 21 (1995), 255 - 269.

[5] M. J. Cowen and R. G. Douglas, Complex geometry and operator theory, Acta Math., 141 (1978), 187 - 261.

[6] M. J. Cowen and R. G. Douglas, Operators possessing an open set of eigenvalues, Functions, series, operators, Vol. I, II (Budapest, 1980), 323 - 341, Colloq. Math. Soc. Janos Bolyai, 35, North-Holland, Amsterdam, 1983.

[7] R. G. Douglas, P. S. Muhly and Carl Pearcy, Lifting Commuting Operators, Michigan Math. Journal, 15 (1968), $385-395$.

[8] M. Dritschel and S. McCullough, The failure of rational dilation on a triply connected domain, J. Amer. Math. Soc., 18 (2005), 873 - 918.

[9] G. Misra, Curvature inequalities and extremal properties of bundle shifts, J. Operator Th., 11 (1984), 305 317.

[10] G. Misra, Completely contractive Hilbert modules and Parrott's example, Acta Math. Hungar., 63 (1994), 291 $-303$.

[11] G. Misra and N. S. N. Sastry, Contractive modules, extremal problems and curvature inequalities, J. Funct. Anal., 88 (1990), 118 - 134

[12] G. Misra and N. S. N. Sastry, Completely contractive modules and associated extremal problems, J. Funct. Anal., 91 (1990), $213-220$.

[13] G. Misra and A. Pal, Contractivity, complete contractivity and curvature inequalities, to appear, Journal d'Analyse Mathematique.

[14] B. Sz.-Nagy and C. Foias, Harmonic analysis of Hilbert space operators, NorthHolland, 1970.

[15] S. Parrott, Unitary dilations for commuting contractions, Pac. J. Math., 34 (1970), 481 - 490.

[16] G. Pisier, An Introduction to the Theory of Operator Spaces, Cambridge University Press, 2003.

[17] V. Paulsen, Completely Bounded Maps and Operator Algebras, Cambridge University Press, 2002.

[18] V. Paulsen, Representations of function algebras, abstract operator Spaces and Banach space geometry, J. Funct. Anal., 109 (1992), 113 - 129.

(G. Misra) Department of mathematics, Indian Institute of Science, Bangalore - 560012 , India

E-mail address, G. Misra: gm@math.iisc.ernet.in

(A. Pal) Department of Mathematics and Statistics, Indian Institute of Science Education And Research Kolkata, Mohanpur - 741246

E-mail address, A. Pal: avijitmath@gmail.com

(C. Varughese) Renaissance Communications, Bangalore - 560058

E-mail address, C. Varughese: cherian@rcpl.com 